\title{
Modelling atmospheric flows with adaptive moving meshes
}

\author{
Christian Kühnlein ${ }^{\mathrm{a}, *}$, Piotr K. Smolarkiewicz ${ }^{\mathrm{b}}$, Andreas Dörnbrack ${ }^{\mathrm{a}}$ \\ ${ }^{a}$ Deutsches Zentrum für Luft- und Raumfahrt (DLR), Institut für Physik der Atmosphäre, 82234 Oberpfaffenhofen, Germany \\ ${ }^{\mathrm{b}}$ National Center for Atmospheric Research, Boulder, CO 80307, USA
}

\section{A R T I C L E I N F O}

\section{Article history:}

Received 7 October 2011

Received in revised form 4 December 2011

Accepted 13 December 2011

Available online 20 December 2011

\section{Keywords:}

Adaptive moving mesh

Multiscale atmospheric flow

Geometric conservation law

Non-oscillatory forward-in-time scheme

\begin{abstract}
A B S T R A C T
An anelastic atmospheric flow solver has been developed that combines semi-implicit nonoscillatory forward-in-time numerics with a solution-adaptive mesh capability. A key feature of the solver is the unification of a mesh adaptation apparatus, based on moving mesh partial differential equations (PDEs), with the rigorous formulation of the governing anelastic PDEs in generalised time-dependent curvilinear coordinates. The solver development includes an enhancement of the flux-form multidimensional positive definite advection transport algorithm (MPDATA) - employed in the integration of the underlying anelastic PDEs - that ensures full compatibility with mass continuity under moving meshes. In addition, to satisfy the geometric conservation law (GCL) tensor identity under general moving meshes, a diagnostic approach is proposed based on the treatment of the GCL as an elliptic problem. The benefits of the solution-adaptive moving mesh technique for the simulation of multiscale atmospheric flows are demonstrated. The developed solver is verified for two idealised flow problems with distinct levels of complexity: passive scalar advection in a prescribed deformational flow, and the life cycle of a large-scale atmospheric baroclinic wave instability showing fine-scale phenomena of fronts and internal gravity waves.
\end{abstract}

(c) 2011 Elsevier Inc. All rights reserved.

\section{Introduction}

In numerical simulation of atmospheric flows, the enormous range of scales (from planetary down to sub-meter) and their interaction poses a formidable challenge. Moreover, density stratification, planetary rotation, and water phase changes increase the complexity of the task. Thanks to the steadily growing computing power and advances in numerical modelling systems, realistic simulations of three-dimensional (3D) atmospheric flows have become standard nowadays. However, the current resources are still insufficient to resolve all relevant multiscale phenomena. For many problems of scientific and public interest, there is a need to increase locally the resolution of contemporary flow solvers by several orders of magnitude.

Currently, the most common approach for the (horizontal) discretisation in established operational and research models of the atmosphere is to employ homogeneous meshes, based on either a grid point/cell or spectral representation in the simulation domain. In contrast, adaptive mesh methods, established in engineering [1], allow to apply finer or coarser mesh sizes in regions of interest. Such techniques offer the possibility to simultaneously resolve targeted local small-scale phenomena and the larger-scale flow. Ideally, only a fraction of the computational expense of a comparable simulation using a globally fine mesh would be required. Static mesh adaptations have been used in atmospheric models for decades [2], with mesh stretching [2] or grid nesting [3] typically applied; latest developments also consider fully unstructured mesh techniques for limited-area modelling as well as global simulations, e.g. [4,5]. However, with static adaptation, the regions of

\footnotetext{
* Corresponding author.

E-mail addresses: christian.kuehnlein@dlr.de (C. Kühnlein), smolar@ucar.edu (P.K. Smolarkiewicz), andreas.doernbrack@dlr.de (A. Dörnbrack).
} 
interests must be known a priori, so the approach is best suited for stationary or quasi-stationary features; e.g. to better resolve flow interaction with topography, or for improving the accuracy of regional forecasts. In general however, static mesh adaptation can be overly restrictive for the simulation of atmospheric phenomena. In particular, large-scale weather systems and associated smaller-scale processes (frontal zones, moist convection, internal gravity waves, etc.) are highly variable in space and time, subject to a continuous process of baroclinic (Rossby) wave growth, decay and propagation, with coexisting smaller-scale processes emerging locally and intermittently. An effective approach to simulate such flows could be a dynamic solution-adaptive re-meshing, with the resolution conforming locally to the flow evolution.

As yet, solution-adaptive mesh methods have not found widespread application in computational meteorology, and there are still a number of open research issues specific to atmospheric flows [6,7]. In particular, it is still unclear how to define suitable mesh refinement criteria (i.e. dynamic criteria that reliably indicate in which regions of the simulation domain a finer or coarser mesh is required), or how to suitably formulate closure models for sub-grid scale processes (such as turbulence and moist convection) on dynamically adapting meshes. Furthermore, it is not clear which of the prevalent adaptation strategies - changing the number of mesh points, moving mesh points, and changing the order of the numerical approximation [7] - as well as their possible combinations, are suitable and effective for atmospheric models. Finally, a solutionadaptive mesh solver must respect inherent balances of hydrostaticity and geostrophy, and has to faithfully represent internal (Rossby and gravity) waves, all of which are canonical aspects of geophysical fluid dynamics.

In [8], the authors presented a generic modelling framework with a deforming mesh capability for the simulation of geophysical flows on scales from micro to planetary. The model's design is based on semi-implicit non-oscillatory forwardin-time (NFT) numerics, optionally integrating a flux-form Eulerian or an advective semi-Lagrangian representation of the non-hydrostatic anelastic equations; ${ }^{1}$ this generic model is known under the name EULAG (Eulerian/semi-Lagrangian fluid solver) [9]. The foundation of the deforming mesh capability in [8] is a time-dependent generalised coordinate transformation, implemented rigorously throughout the governing PDEs. First test applications were presented in [8] that demonstrated the viability of the model to simulate atmospheric flows using adaptive meshes. However, the applications did not exploit the full capacity of the underlying generalised coordinate framework. The moving mesh adaptation was prescribed (a priori) only for well-defined targeted regions, adopting either analytical stretching functions or a single nested mesh of a given size. In particular, there was no dynamic interaction between the solution of the anelastic model and the mesh adaptation.

Here, we build upon the work of [8] by consistently extending their modelling framework with a general solution-adaptive mesh capability. In particular, an anelastic flux-form NFT dynamical core is augmented with so-called moving mesh partial differential equations (MMPDEs) [10] for the solution-adaptive mesh generation. The idea of moving mesh methods (i.e. r-adaptivity) is to redistribute a fixed number of mesh nodes for adaptation in the physical space, where the actual problem is posed, though retaining virtues of Cartesian (structured) grid calculations in the transformed computational domain, where the problem is solved. Advantages are efficient numerical calculations and low memory requirements. In addition, moving mesh methods are ideally suited for large-scale parallel computing architectures. ${ }^{2}$

Advective transport in our modelling framework is based on MPDATA (for "multidimensional positive definite advection transport algorithm," see [12,13] and references therein). MPDATA schemes belong to the class of nonlinear high-resolution methods [14] that offer solutions free of spurious oscillations, while maintaining higher-order accuracy away from discontinuities for arbitrary flows. The particular nonlinear design of MPDATA [14] makes it a viable advection method for implicit large-eddy simulation (ILES) of high-Reynolds number turbulent flows [15,14,16,17]. In ILES, the large-scale turbulent motions are simulated explicitly, while the subgrid-scale modelling of turbulence is left to the self-adaptive dissipative nature of the truncation terms in the advection scheme. This is in contrast to the standard LES approach, where the effect of the subgrid-scale dynamics on the resolved scales is treated with explicit sub-grid scale models [18,19]. An ILES option is of special relevance to atmospheric flow simulations with adaptive meshing, where explicit modelling of subgrid-scale turbulence is difficult. Furthermore, the non-oscillatory character of the solution scheme per se represents an important ingredient of an accurate and robust mesh adaptation algorithm, especially when the mesh refinement is driven by the solution itself. In terms of the latter, it is added that MPDATA's special iterative error-reducing design can be exploited to derive a posteriori refinement indicators for mesh adaptation [20].

A crucial aspect for the efficacy of an adaptive mesh solver is the quality of the generated mesh. In terms of the solutionadaptive mesh generation apparatus applied in this work, the mesh quality depends primarily on the specification of the socalled monitor function in the MMPDEs. Yet, it is unclear how to effectively specify monitor functions in such complex, multiscale flows like in climate or weather prediction. Therefore, systematic research is needed to address this aspect. Another important aspect of the adaptive mesh solver is to ensure that qualities of the numerics proven for standard grids are maintained under the solution-adaptive moving meshes. Such qualities include the formal order of accuracy, conservation, and the preservation of physical extrema (i.e. the non-oscillatory property [21]). Herein, the governing flow equations are cast in a conservation form. Solving advection transport equations in conservation form signifies the consistency/compatibility of the applied advection solver with the associated discrete mass conservation law - hereafter referred to as compatibility - important for preventing spurious extrema [22-24]. When considering a formulation in time-dependent coordinates,

\footnotetext{
1 Anelastic, sound-proof equations represent analytically filtered subsets of the fully compressible equations for density-stratified geophysical/astrophysical flows that do not contain the fast energetically insignificant acoustic modes yet capture thermal aspects of compressibility.

${ }^{2}$ Favourable parallelisation characteristics are essential in the area of atmospheric flow modelling, where the use of hundreds to tens of thousands of processors has become reality nowadays [9,11].
} 
the mass conservation law takes on a compressible form although the physical flow addressed is assumed to be incompressible or anelastic. This complicates the integration, as an accuracy issue emerges due to the time-dependent mesh geometry that is not accounted for in the standard numerical scheme for static coordinates. The subject is known under the term geometric conservation law (GCL) $[25,26]$. The importance of satisfying the GCL on the level of discretisation has been repeatedly discussed in literature, e.g. [25,27-29]. A common approach originally proposed in [25] is to incorporate the GCL as an auxiliary prognostic equation for the evolution of the mesh cell volumes. In the present work, the issue of satisfying the GCL is addressed differently. In the spirit of the mass conservation equation in incompressible/anelastic models, it is proposed to treat the GCL as an elliptic problem.

The paper is organised as follows. Section 2 reviews the analytical and numerical formulation of the anelastic modelling framework underlying the present work. Section 3 gives a summary of the apparatus that is employed for the generation of the solution-adaptive moving mesh in our model. Section 4 elaborates on the compatibility of the flux-form NFT integration of the transformed anelastic equations under solution-adaptive moving meshes. Substantiating test simulations are provided for a two-dimensional scalar advection problem in Section 5. Finally in Section 6, the capability of the developed solutionadaptive moving mesh NFT flow solver is investigated by means of an archetype multiscale atmospheric flow problem, a three-dimensional life cycle of a dry baroclinic instability on the $f$-plane.

\section{Modelling framework}

This section reviews the anelastic modelling framework for the implementation of the solution-adaptive moving mesh NFT solver developed in this work. Here, only the "dry", adiabatic, inviscid version of our model is presented. Complexities that arise from the inclusion of moist processes, diabatic and viscous effects $[30,31,17]$ are disregarded for simplicity of the presentation. The focus is on the essential aspects of formulating and integrating the anelastic equations in generalised coordinates.

\subsection{Anelastic flow equations in generalised coordinates}

Following the notation of [8], let $\mathbf{S}_{p}$ denote the physical space where the problem is posed - restricted to be Cartesian in the present work, notwithstanding the generality of the development in [8]. An irregular, possibly time-dependent, subdomain $\mathcal{D}_{p} \subseteq \mathbf{S}_{p}$ with an assumed tuple of coordinates $(t, \mathbf{x}) \equiv(t, x, y, z)$ in $\mathbf{S}_{p}$ can be mapped into a regular computational subdomain $\mathcal{D}_{t} \subseteq \mathbf{S}_{t}$ in a transformed space $\mathbf{S}_{t}$ with its own tuple of generalised coordinates $(\bar{t}, \overline{\mathbf{x}}) \equiv(\bar{t}, \bar{x}, \bar{y}, \bar{z})$ :

$$
(\bar{t}, \overline{\mathbf{x}}) \equiv(t, \mathcal{F}(t, \mathbf{x})): \mathcal{D}_{p} \rightarrow \mathcal{D}_{t} .
$$

The generic mapping (1) is understood here to be a diffeomorphism, i.e. $\mathcal{F}(t, \mathbf{x})$ is a bijective map between manifolds $\mathcal{D}_{p}$ and $\mathcal{D}_{t}$ that is at least $C^{2}$-continuously differentiable. ${ }^{3}$ Rather than solving the physical problem posed with the variable adaptive mesh in $\mathbf{S}_{p}$, it is transformed according to the mapping (1) and discretised on a fixed regular mesh in $\mathbf{S}_{t}$. Using a tensorial description, the non-hydrostatic anelastic equations of Lipps and Hemler [32] in a strong conservation formulation (see Introduction in [33] for a discussion) can be written with respect to $\mathbf{S}_{t}$ as

$$
\begin{aligned}
& \frac{\partial\left(\rho^{*} v^{j}\right)}{\partial \bar{t}}+\frac{\partial\left(\rho^{*} \bar{v}^{*^{k}} v^{j}\right)}{\partial \bar{x}^{k}}=-\rho^{*} \widetilde{G}_{j}^{k} \frac{\partial \pi^{\prime}}{\partial \bar{x}^{k}}+\rho^{*} g \frac{\theta^{\prime}}{\theta_{b}} \delta_{3}^{j}-\rho^{*} \varepsilon_{j i k} f_{i} v^{\prime k}-\rho^{*} \alpha_{M} v^{j} \\
& \frac{\partial\left(\rho^{*} \theta^{\prime}\right)}{\partial \bar{t}}+\frac{\partial\left(\rho^{*} \bar{v}^{*^{k}} \theta^{\prime}\right)}{\partial \bar{x}^{k}}=-\rho^{*} \bar{v}^{s^{k}} \frac{\partial \theta_{e}}{\partial \bar{x}^{k}}-\rho^{*} \alpha_{H} \theta^{\prime} \\
& \frac{\partial\left(\rho^{*} \bar{v}^{s^{k}}\right)}{\partial \bar{x}^{k}}=0
\end{aligned}
$$

where $i, j, k=1,2,3$; and the Einstein summation convention applies, unless otherwise stated. The Kronecker delta $\delta_{3}^{j}$ in the buoyancy term of the momentum Eq. (2a) is 1 for $j=3$, and 0 for $j \neq 3$. The Levi-Civita symbol $\varepsilon_{j i k}$ [34] in the Coriolis term represents the vector product.

As far as the physical aspects of the system (2) are concerned, $v^{j}, \theta, \rho$, and $\pi$, denote, respectively, the velocity components with respect to $\mathbf{S}_{p}$, potential temperature, density, and a density-normalised pressure. The symbol $g$ represents the gravitational acceleration, $f_{i}$ stands for the components of the Coriolis parameter arising due to the global rotation of the domain (see Appendix A in [8]). The subscript $b$ appearing with $\theta_{b}$ in the buoyancy term of (2a) refers to the basic state, a prescribed horizontally-homogeneous hydrostatic reference state, characteristic of the anelastic approximation, cf. [3]. In addition to the formal basic state, a more general ambient (also called environmental) state, that can vary in the vertical as well as the horizontal direction, is denoted with the subscript $e$, and defined to satisfy a balanced subset of the system (2). The reader is referred to [35,9] for a discussion of the ambient states and their benefits. All primed variables $\theta^{\prime}, v^{\prime}$, and $\pi^{\prime}$ in (2) correspond to deviations from the ambient state. An additional aspect of the system (2) are Rayleigh-type forces with relaxation parameters $\alpha_{M}$ and $\alpha_{H}$ employed to simulate (internal) wave-absorbing layers near the boundaries of the model domain [36].

\footnotetext{
${ }^{3}$ Notably, the numerical formulation of the model allows for discontinuities in the coordinate mapping to mimic nested grids, see [8] for an example.
} 
A generalised density $\rho^{*} \equiv \rho_{b} \bar{G}$ is conveniently introduced in (2) as the product of the basic state density $\rho_{b}$ and the Jacobian $\bar{G}$ of the implemented coordinate transformation. For the sake of clarity, $\rho^{*}$ represents a positive variable that incorporates the effects of both, the physical volumetric variation due to background stratification contained in the timeindependent prescribed reference density $\rho_{b}$, plus changes of the geometric volume through the Jacobian $\bar{G}$ of the underlying coordinate transformation, that may be time-dependent.

Furthermore, $\bar{v}^{*^{k}}:=d \bar{x}^{k} / d \bar{t}$ is the contravariant velocity in the transformed system $\mathbf{S}_{t}$. The velocity $\bar{v}^{s^{k}}$ occurring in the entropy (2b) and continuity (2c) equations is the so-called solenoidal velocity [37] defined as

$$
\bar{v}^{s^{k}}:=\bar{v}^{*^{k}}-\frac{\partial \bar{x}^{k}}{\partial t}=: \bar{v}^{*^{k}}-\bar{v}^{g^{k}}
$$

The rightmost term in (3) defines the mesh velocity $\bar{v}^{\mathrm{g}^{k}}$ in $\mathbf{S}_{t}$. A transformation that relates the physical and the solenoidal velocity directly is given as

$$
\bar{v}^{s^{k}}=\widetilde{G}_{j}^{k} \nu^{j}
$$

The symbol $\widetilde{G}_{j}^{k}:=\partial \bar{x}^{k} / \partial x^{j}$ appearing in (4) and in the pressure gradient term of (2a), denotes the elements of the Jacobian matrix.

Underlying the model formulation in the generalised coordinates are fundamental tensor identities, e.g. [28,31,38], where at least two deserve brief consideration here. Among these is the Kronecker delta identity

$$
\delta_{s}^{r} \equiv \frac{\partial \bar{x}^{r}}{\partial x^{q}} \frac{\partial x^{q}}{\partial \bar{x}^{s}}
$$

where $q, r, s=0,1,2,3, \bar{t} \equiv \bar{x}^{0}, t \equiv x^{0}$; which states the reciprocity of the covariant and contravariant base vectors describing the system $\mathbf{S}_{t}$. Given the computed metric coefficients $\partial x^{q} / \partial \bar{x}^{s}$ in the model computational space $\mathbf{S}_{t}$, the identity (5) provides the relationships to determine the inverse metric coefficients $\partial \bar{x}^{r} / \partial x^{q}$. Another identity that arises naturally with the flow equations in conservation form is the multi-component tensor geometric conservation law (GCL)

$$
\frac{1}{\bar{G}} \frac{\partial}{\partial \bar{x}^{r}}\left(\bar{G} \frac{\partial \bar{x}^{r}}{\partial x^{s}}\right) \equiv 0
$$

The GCL (6) represents a compact differential statement about the conservation of space, under the general mapping of the coordinates (1). For $s=1,2,3$ it describes the surface conservation for a closed volume cell, and relates the three-dimensional spatial variation of the inverse metric coefficients and the Jacobian determinant. For $s=0$, the GCL (6) describes the conservation of volume (proportional to $\bar{G})$ according to $\bar{G}^{-1}\left[\partial \bar{G} / \partial \bar{t}+\partial / \partial \bar{x}^{k}\left(\bar{G} \partial \bar{x}^{k} / \partial t\right)\right] \equiv 0$, i.e. it relates, in the transformed space $\mathbf{S}_{t}$, the divergence of the fluxes of volume variation in space with the changes of volume variation in time. The importance of satisfying the GCL on the level of discretisation in the solution of conservation laws was already emphasised in [25].

The utilised form of the mass continuity Eq. (2c) follows from the most general tensor-invariant representation of mass continuity under the time-dependent transformation (1). The latter reads

$$
\frac{1}{\bar{G}} \frac{\partial\left(\rho^{*} \bar{v}^{*^{r}}\right)}{\partial \bar{x}^{r}}=0
$$

with $r=0,1,2,3, \bar{v}^{*^{0}} \equiv 1$; upon which (7) can be displayed as $\bar{G}^{-1}\left[\partial \rho^{*} / \partial \bar{t}+\partial\left(\rho^{*} \bar{v}^{*^{k}}\right) / \partial \bar{x}^{k}\right]=0$. However, under the assumption that the basic state density is stationary $\rho_{b}=\rho_{b}(\mathbf{x})$, and from the GCL identity (6), the form (7) is analytically equivalent to (2c) [37]. This allows to employ the anelastic form (2c) instead of the elastic form (7) in the governing system (2). As a result, it simplifies the design of the numerical model (see Section 2.2), and improves the efficacy of the solution procedure in timedependent generalised coordinates. Notwithstanding the use of (2c) in the system (2), it is demonstrated in Section 4 that the general form of the mass continuity Eq. (7) has important implications for the design and implementation of the advective flux-form NFT integration under moving meshes.

\subsection{Numerical solution procedure}

Each prognostic equation of the anelastic system (2) can be written as the following generic Eulerian conservation law

$$
\frac{\partial\left(\rho^{*} \psi\right)}{\partial \bar{t}}+\bar{\nabla} \cdot\left(\rho^{*} \overline{\mathbf{v}}^{*} \psi\right)=\rho^{*} R^{\psi}
$$

where $\psi=\psi(\bar{t}, \overline{\mathbf{x}})$ denotes the transported mass-specific variable (i.e. $\psi$ represents the three components of the physical velocity vector $\mathbf{v}$ and the potential temperature perturbation $\left.\theta^{\prime}\right), R^{\psi}$ subsumes the appendant source terms, $\overline{\mathbf{v}}^{*} \equiv d \overline{\mathbf{x}} / d \bar{t}$ is the contravariant velocity vector in $\mathbf{S}_{t}$, and the nabla operator $\bar{\nabla} \equiv \partial / \partial \overline{\mathbf{x}}$ denotes the vector of partial derivatives with respect to the generalised coordinates $\overline{\mathbf{x}}$ in the computational space $\mathbf{S}_{t}$. Following [39,12], a compact description of the solution algorithm on the regular computational mesh $\left(\bar{t}^{n}, \overline{\mathbf{x}}_{\mathbf{i}}\right)$ can be written as

$$
\psi_{\mathbf{i}}^{n+1}=\mathcal{A}_{\mathbf{i}}(\tilde{\psi})+\left.0.5 \delta \bar{t} R^{\psi}\right|_{\mathbf{i}} ^{n+1},
$$


where $\mathcal{A}_{\mathbf{i}}$ symbolises a NFT advection transport scheme, and $\tilde{\psi} \equiv \psi^{n}+\left.0.5 \delta \bar{t} R^{\psi}\right|^{n}$. The $n, n+1$ superscripts denote the time level, the subscript $\mathbf{i}$ denotes the spatial mesh vector index, and $\delta \bar{t}=\bar{t}^{n+1}-\bar{t}^{n}$ is the time step increment. In our model, $\mathcal{A}$ integrates the homogeneous conservation law (8) employing the fully second-order-accurate multidimensional advection transport algorithm MPDATA (Section 4). Advecting the auxiliary field $\tilde{\psi}$ (instead of the variable $\psi^{n}$ alone) compensates for $\mathcal{O}(\delta \bar{t})$ truncation errors proportional to the divergence of the advective flux of the source terms $R^{\psi}$ [39].

The template algorithm (9) for (2) represents a system that is fully implicit with respect to the dependent variables $\mathbf{v}$, $\pi^{\prime}$ and $\theta^{\prime}$, with the explicit complement composed of $\hat{\mathbf{v}}:=\mathcal{A}(\tilde{\mathbf{v}})$ and $\hat{\theta}^{\prime}:=\mathcal{A}\left(\tilde{\theta}^{\prime}\right)$. In particular, for the momentum Eq. (2a), the algorithm (9) results in

$$
\mathbf{v}_{\mathbf{i}}=\hat{\mathbf{v}}_{\mathbf{i}}-0.5 \delta \bar{t}\left(\widetilde{\mathbf{G}} \bar{\nabla} \pi^{\prime}\right)_{\mathbf{i}}+0.5 \delta \bar{t} \mathbf{F}_{\mathbf{i}}\left(\mathbf{v}, \hat{\theta}^{\prime}\right)
$$

with

$$
\mathbf{F}_{\mathbf{i}}\left(\mathbf{v}, \hat{\theta}^{\prime}\right) \equiv-\left(\frac{\mathbf{g}}{\theta_{b}} \frac{\hat{\theta}^{\prime}-0.5 \delta \bar{t}\left(\left(\widetilde{\mathbf{G}}^{T} \mathbf{v}\right) \cdot \bar{\nabla} \theta_{e}\right)}{1+0.5 \delta \bar{t} \alpha_{H}}\right)_{\mathbf{i}}-\left(\mathbf{f} \times \mathbf{v}^{\prime}\right)_{\mathbf{i}}-\left(\alpha_{M} \mathbf{v}^{\prime}\right)_{\mathbf{i}}
$$

accounting for the implicit treatment of buoyancy via the entropy Eq. (2b). ${ }^{4}$ All superscripts indicating the $n+1$ time level have been dropped here, since there is no ambiguity. The symbol $\widetilde{\mathbf{G}}$ that appears in the utilised compact description, is identified with the Jacobian matrix $\widetilde{G}_{j}^{k}$, defined in Section 2.1. Organising (10) into explicit and implicit parts gives

$$
\mathbf{v}_{\mathbf{i}}=\hat{\mathbf{v}}_{\mathbf{i}}-0.5 \delta \bar{t}\left(\widetilde{\mathbf{G}} \bar{\nabla} \pi^{\prime}\right)_{\mathbf{i}}+0.5 \delta \widetilde{\mathbf{t}}_{\mathbf{i}}(\mathbf{v}),
$$

where $\hat{\hat{\mathbf{v}}}$ subsumes all explicitly known terms, and $\widetilde{\mathbf{F}}$ denotes the implicit remainder of (11), i.e. the linear homogeneous operator acting on v. Provided that the underlying grid is co-located with respect to all prognostic variables, ${ }^{5}$ algebraic inversion of (12) leads to a unique expression for the vector $\mathbf{v}_{\mathbf{i}}$. Subsequent insertion into (4) gives an expression for the solenoidal velocity $\left.\overline{\mathbf{v}}^{s} \equiv \widetilde{\mathbf{G}}^{T} \tilde{\tilde{\mathbf{v}}}-(\mathbf{I}-0.5 \delta \bar{t} \widetilde{\mathbf{F}})^{-1}\left(\widetilde{\mathbf{G}} \bar{\nabla} \pi^{\prime \prime}\right)\right]$, where $\tilde{\tilde{\mathbf{v}}}:=(\mathbf{I}-0.5 \delta \bar{t} \widetilde{\mathbf{F}})^{-1} \hat{\hat{\mathbf{v}}}$ and $\pi^{\prime \prime}:=0.5 \delta \bar{t} \pi^{\prime}$. Eventually, the solenoidal velocity $\overline{\mathbf{v}}^{s}$ is substituted into the anelastic mass continuity Eq. (2c) to obtain an elliptic equation for $\pi^{\prime \prime}$

$$
\left\{-\frac{\delta \bar{t}}{\rho^{*}} \bar{\nabla} \cdot\left(\rho^{*} \widetilde{\mathbf{G}}^{T}\left[\tilde{\mathbf{v}}-(\mathbf{I}-0.5 \delta \bar{t} \widetilde{\mathbf{F}})^{-1}\left(\widetilde{\mathbf{G}} \bar{\nabla} \pi^{\prime \prime}\right)\right]\right)\right\}_{\mathbf{i}}=0,
$$

a complete development of which is given in Appendix A of [8]. The applied normalisation by $\left(\delta \bar{t} / \rho^{*}\right)$ gives the residual errors of (13) the meaning of the divergence of a dimensionless velocity on the grid. The latter compares directly to the magnitude of the Courant numbers, and facilitates the specification of physically meaningful accuracy thresholds $\left\|\left(\delta \bar{t} / \rho^{*}\right) \bar{\nabla} \cdot\left(\rho^{*} \overline{\mathbf{v}}^{s}\right)\right\|<\epsilon[40]$. Moreover, multiplication by the factor $(-1)$ assures the formal negative-definiteness of the elliptic operator [8]; whereas the scaling by $\left(1 / \rho^{*}\right)$ acts as a diagonal preconditioner, important for deep atmospheres where $\rho^{*}$, respectively $\rho_{b}$, can vary several orders of magnitude over the vertical depth of the simulation domain [36]. Dirichlet boundary conditions along $\partial \mathcal{D}_{t}$ prescribed on the normal component of the solenoidal velocity $\overline{\mathbf{v}}^{s} \cdot \overline{\mathbf{n}}$, which are subject to the integrability condition $\int_{\partial \mathcal{D}_{t}} \rho^{*} \overline{\mathbf{v}}^{s} \cdot \overline{\mathbf{n}} d \bar{\sigma}=0$, imply the correct Neumann boundary conditions for $\pi^{\prime \prime}$ $[8,33]$. A preconditioned generalised conjugate residual GCR scheme $[41,42]$ is employed to solve the formulated elliptic boundary value problem (13). The solution to (13) produces the updated solenoidal velocity. From this, the updated physical and contravariant velocity components are obtained using the relations (4) and (3), respectively. After completing the velocity solution, the updated $\theta^{\prime}$ follows readily as

$$
\theta^{\prime}=\frac{\hat{\theta}^{\prime}-0.5 \delta \bar{t} \overline{\mathbf{v}}^{s} \cdot \bar{\nabla} \theta_{e}}{1+0.5 \delta \bar{t} \alpha_{H}} .
$$

\section{Moving mesh apparatus}

The solution adaptivity of the mesh in our model is based on the moving mesh technique. The latter typically resorts to the concept of continuous coordinate transformations for the mesh generation [43]: Given the fixed regular mesh on the computational domain $\mathcal{D}_{t}$ as a reference, the time-dependent irregular adaptive mesh on the physical domain $\mathcal{D}_{p}$ is described by means of a mapping $\mathcal{D}_{t} \rightarrow \mathcal{D}_{p}$. In the present work, a two-dimensional horizontal adaptive mesh is considered. The specific form of the mapping underlying the mesh generation apparatus is

$$
(x, y) \equiv(x(\bar{t}, \bar{x}, \bar{y}), y(\bar{t}, \bar{x}, \bar{y})): \mathcal{D}_{t}^{h} \rightarrow \mathcal{D}_{p}^{h}
$$

where $\mathcal{D}_{t}^{h}$ and $\mathcal{D}_{p}^{h}$ denote the two-dimensional horizontal subdomains of the computational domain $\mathcal{D}_{t}$ and physical domain $\mathcal{D}_{p}$, respectively. By definition, the computational domain $\mathcal{D}_{t}^{h}$ denotes a unit square.

Methods of grid generation based on a mapping of coordinates often rely on a variational approach. It allows to impose various constraints (e.g. adaptivity with respect to a refinement indicator, smoothness, orthogonality) on the generated

\footnotetext{
4 The gravity vector in (11) is given as $\mathbf{g}=(0,0,-g)^{T}$.

5 Arakawa A- or B-grids are possible options [36].
} 
mesh, e.g. [44]. The task is to choose appropriately defined functionals that incorporate the desired properties. Then, optimisation leads to a "compromise" mesh that combines the various properties involved. Solutions of the corresponding elliptic Euler-Lagrange equations provide the mesh mapping. Following [45,10], a system of nonlinear Euler-Lagrange equations is derived from a generic functional ${ }^{6}$ and augmented to a parabolic form, for efficient numerical solution. The resulting MMPDEs for the mapping (15) are given as

$$
P\left(\mathbf{x}_{h}, M\right) \frac{\partial \mathbf{x}_{h}}{\partial \bar{t}}=\sum_{i, j=1,2} D_{i j}\left(\mathbf{x}_{h}, M\right) \frac{\partial^{2} \mathbf{x}_{h}}{\partial \bar{x}^{i} \partial \bar{x}^{j}}+\sum_{i=1,2} C_{i}\left(\mathbf{x}_{h}, M\right) \frac{\partial \mathbf{x}_{h}}{\partial \bar{x}^{i}}
$$

with the coefficients

$$
\begin{aligned}
& D_{i j}\left(\mathbf{x}_{h}, M\right)=\nabla_{h} \bar{x}^{i} \cdot M^{-1} \nabla_{h} \bar{x}^{j}, \quad C_{i}\left(\mathbf{x}_{h}, M\right)=-\nabla_{h} \bar{x}^{i} \cdot\left(\sum_{k=1,2} \frac{\partial M^{-1}}{\partial \bar{x}^{k}} \nabla_{h} \bar{x}^{k}\right), \\
& P\left(\mathbf{x}_{h}, M\right)=\mathcal{T} \sqrt{\left(D_{11}\right)^{2}+\left(D_{22}\right)^{2}+\left(C_{1}\right)^{2}+\left(C_{2}\right)^{2}},
\end{aligned}
$$

where $M\left(t, \mathbf{x}_{h}\right)$ is the monitor function, a $2 \times 2$ symmetric positive definite matrix for describing the structure (and adaptivity) of the mesh, while $\mathbf{x}_{h} \equiv(x, y)^{T}$ and $\nabla_{h} \equiv \partial / \partial \mathbf{x}_{h}$. The symbol $\mathcal{T}$ in (16) represents a mesh relaxation time $(\mathcal{T}>0)$ which is employed as a smoothing parameter of the mesh motion. The smaller (larger) $\mathcal{T}$, the faster (slower) reacts the mesh to changes of the monitor function.

The two MMPDEs (16) require the specification of appropriate boundary conditions. At non-periodic boundaries (e.g. open boundaries or rigid walls) of the physical model (2), Dirichlet-type conditions are specified for $\mathbf{x}_{h}$. MMPDEs for the distribution of the mesh points $\mathbf{x}_{h}$ on individual segments $\Gamma_{p}$ of the boundary $\partial \mathcal{D}_{p}^{h}$ (typically between fixed corner points) are solved prior to (16) at every time step. The one-dimensional analogue of (16) reads

$$
p(s, \mu) \frac{\partial s}{\partial \bar{t}}=\mu \frac{\partial^{2} s}{\partial \bar{s}^{2}}+\frac{\partial \mu}{\partial \bar{s}} \frac{\partial s}{\partial \bar{s}},
$$

where the arc-length coordinates $s$ and $\bar{s}$ parameterise the physical $\Gamma_{p}$ and the associated computational $\Gamma_{t}$ boundary (line) segments, respectively. The scalar-valued monitor function $\mu$ in (17) is computed as the projection $\mu(t, s)=\mathbf{t}^{T} M \mathbf{t}$ of the matrix-valued monitor function $M$ along the boundary $\partial \mathcal{D}_{p}^{h}$, where $\mathbf{t}$ is the local tangent vector to $\partial \mathcal{D}_{p}^{h}$. The coefficient $p(s, \mu)$ in (17) is given as $p(s, \mu)=\mathcal{T}\left[\mu^{2}+(\partial \mu / \partial \bar{s})^{2}\right]^{1 / 2}$. Periodic boundaries are another choice in the physical model (2), which are thoroughly implemented in the solution scheme of the MMPDEs (16).

The specific form of $M$ applied in the present work is given as a diagonal matrix

$$
M=q I,
$$

where $I$ denotes the $2 \times 2$ identity matrix and $q=q\left(t, \mathbf{x}_{h}\right)$ a strictly positive scalar weighting function for the mesh density [48]. Given a mesh refinement indicator $\Phi\left(t, \mathbf{x}_{h}\right) \geqslant 0$, i.e. some measure for the local error in the flow computation that indicates where a finer or coarser mesh is required, the specification for $q$ that is adopted here is

$$
q\left(t, \mathbf{x}_{h}\right)=1+\frac{\beta}{1-\beta} \frac{\Phi}{\langle\Phi\rangle_{h}},
$$

see [10,49]. The symbol $\langle\Phi\rangle_{h}$ in (19) defines the average of $\Phi$ over the horizontal domain $\mathcal{D}_{p}^{h}$. The parameter $0 \leqslant \beta<1$ in (19) allows user-specified control over the strength of the adaptation [10]. It is common practice to filter the weighting function $q$ in order to obtain a smoother mesh and, eventually, to improve the solution properties of the model, e.g. [10,50,51]. Here, a standard Gaussian filter as given in [51] is used. Typical numbers of filter passes in the present solver are 8 to 15.

Solution-adaptivity of the mesh in the framework of the anelastic solver presented in Section 2 is accomplished through an algorithmic coupling with the MMPDE apparatus. The adopted solution approach integrates the physical set of anelastic Eqs. (2) and the coupled MMPDEs (16) alternately for each time step. Unlike in [10,49], the MMPDEs are integrated with the same time step $\delta \bar{t}$ as the physical model (2). In particular, no substeps of the MMPDE integration are applied for the simulation problems considered in the present work. The principal requirements for the numerical solution of the MMPDEs (16) are robustness and efficiency. The formal accuracy of the mesh computation is of minor concern because it is not a determining factor for the accuracy of the obtained physical solution computed on the adaptive mesh. Let $\mathcal{L}$ be the spatial difference operator for the rhs of the MMPDEs (16), the implemented temporal discretisation is the backward Euler scheme $P\left(\mathbf{x}_{h}^{n+1}-\mathbf{x}_{h}^{n}\right) / \delta \bar{t}=\mathcal{L}\left(\mathbf{x}_{h}^{n+1}\right)$, implicit for the dependent variable $\mathbf{x}_{h}^{n+1}$. In the model implementation, the coefficients $D_{i j}$ and $C_{i}$ in (16) are frozen at the time level $n$, thereby circumventing the nonlinearity of the problem (16). Standard central differences are used to discretise all spatial partial derivatives. Given either Dirichlet or periodic boundary conditions for $\mathbf{x}_{h}$, the resulting algebraic problem is solved by a generalised conjugate residual GCR scheme [41,42]. As a stopping criterion, the difference between successive GCR iterative solutions $\phi^{v}$ are related to the extensions $L_{x}$ and $L_{y}$ of the physical domain $\mathcal{D}_{p}$ according to

\footnotetext{
${ }^{6}$ Here, the functional is formulated in $\mathbf{S}_{p}$, and the resulting Euler-Lagrange equations are transformed into $\mathbf{S}_{t}$ for the model implementation. Compared to a functional directly formulated in $\mathbf{S}_{t}$, this procedure provides a more robust mesh generator $[10,46,47]$.
} 


$$
\forall \phi=x, y \quad\left\|\phi^{v+1}-\phi^{v}\right\|_{\infty}<\epsilon \min \left(L_{x}, L_{y}\right) .
$$

A value of $\epsilon=10^{-7}$ in (20) has been employed successfully throughout all experiments in this work. ${ }^{7}$ The described MMPDE $^{2}$ solution procedure is fully embedded in the parallel coding structure of our anelastic solver. The discretisation given above applied to the one-dimensional boundary MMPDEs (17) results in a tridiagonal system of algebraic equations, which is readily solved by the standard Thomas algorithm.

\section{Flux-form NFT integration under moving meshes}

This section elaborates on the design of flux-form NFT advection schemes in time-dependent generalised coordinates. The subject is the effective combination of the advective integration with solution-adaptive moving meshes generated numerically by the MMPDE apparatus of Section 3, rather than being prescribed by smooth analytical functions [8,33,29] that tend to have lower demands on integration schemes. While the ideas presented are of relevance to the general class of flux-form forward-in-time (FT) schemes, the emphasis here is on the particular advection scheme MPDATA [12,13].

\subsection{Introductory considerations}

The advective scalar transport equation in conservation form

$$
\frac{\partial\left(\rho^{*} \psi\right)}{\partial \bar{t}}+\bar{\nabla} \cdot\left(\rho^{*} \overline{\mathbf{v}}^{*} \psi\right)=0
$$

is considered, i.e. the homogeneous version of ( 8 ). The mass conservation law associated with (21) is found by setting $\psi \equiv 1$

$$
\frac{\partial \rho^{*}}{\partial \bar{t}}+\bar{\nabla} \cdot\left(\rho^{*} \overline{\mathbf{v}}^{*}\right)=0
$$

cf. (7). Eq. (22) will be referred to as the generalised anelastic mass conservation law (GMCL) in the subsequent discussion. The combination of (21) and (22) amounts to the evolution equation $d \psi / d \bar{t}=0$ (where $d / d \bar{t}=\partial / \partial \bar{t}+\overline{\mathbf{v}}^{*} . \bar{\nabla}$ ), which specifies the invariance of $\psi$ along the trajectories of the contravariant flow field $\overline{\mathbf{v}}^{*}$. Specifically, it conveys that advection with $\overline{\mathbf{v}}^{*}$ can change neither the sign nor the extrema of the transported variable $\psi$ [13]. One can manipulate (21) into the Lagrangian form without explicitly assuming the validity of the GMCL (22)

$$
\frac{d \psi}{d \bar{t}}=-\frac{\psi}{\rho^{*}}\left(\frac{\partial \rho^{*}}{\partial \bar{t}}+\bar{\nabla} \cdot\left(\rho^{*} \overline{\mathbf{v}}^{*}\right)\right),
$$

which shows that inaccuracies associated with the numerical implementation of the GMCL (22) result in spurious forces for $\psi$ in (21) along the trajectories in the flow field $\overline{\mathbf{v}}^{*}$. The preceding explains that the GMCL (22) entails a compatibility condition [23] on the solution scheme for (21).

In the following, a detailed analysis of the integration of (21) using the NFT scheme MPDATA is conducted. Two properties of the integration are considered. First, the property of the advection scheme to reduce exactly to the associated discrete form of the GMCL (22) is investigated. The condition to be met for this consistency is that an initially uniform field $\psi$ (e.g. $\psi \equiv 1$ ) is exactly maintained by the scheme. This property is typically referred to as constancy preservation, e.g. [52]. The constancy preservation is straightforwardly satisfied under stationary coordinates but it requires a modification of the error-compensative, "antidiffusive" pseudo-velocities in MPDATA under general non-stationary coordinates, which is proposed here. Secondly, it is investigated whether the discrete implementation of the GMCL (22) is itself satisfied in the integration. In the modelling framework presented in Section 2, the anelastic mass conservation is explicitly implemented for the solenoidal velocity $\overline{\mathbf{v}}^{s}$, thereby using the (computationally) advantageous "incompressible" form (2c) in the system (2). By adopting this formulation, the analytic identity

$$
\frac{\partial \rho^{*}}{\partial \bar{t}}+\bar{\nabla} \cdot\left(\rho^{*} \overline{\mathbf{v}}^{g}\right) \equiv 0
$$

which represents the residual term between (22) and (2c), is implicitly invoked [37]. For a uniform basic-state density $\rho_{b}$, (24) is equivalent to the time-component $(s=0)$ of the GCL identity (6). Hence, (24) can be considered as a generalised GCL for the anelastic system. Clearly, the formulation of the anelastic model in time-dependent generalised coordinates must ensure that the GCL (24) is satisfied on the level of discretisation in order to provide an accurate scalar transport solution to (21). In particular, it must be ensured that possible errors in the representation of (24) appear small in (23) compared to residual errors from (2c) or compared to physical forcings included when considering the generic form (8), see [40] for a related discussion. Here, the issue of satisfying the GCL in NFT schemes under solution-adaptive moving meshes is treated thoroughly. As a particular feature, the incorporation of a diagnostic approach for the treatment of the GCL (24) as an elliptic problem is developed. The proposed diagnostic approach can effectively be applied to satisfy the GCL (24) - and therefore the GMCL (22) - under general solution-adaptive moving meshes.

\footnotetext{
7 The combination of (20) with a criterion based on a residual error norm is required because (20) can fail if the iterative solver stalls.
} 
As will be demonstrated by means of numerical experiments, if the two properties of the solution scheme given above are not fulfilled, significant errors may occur in the MPDATA solution of (21) under solution-adaptive moving meshes. In particular, it is shown that both properties are required not only to preserve a uniform scalar field $\psi_{c} \neq 0$, but also to retain the more general non-oscillatory/shape-preserving character of the scheme and hence to avoid the creation of non-physical extrema. The latter can occur despite the non-oscillatory enhancement of MPDATA [21]; however, the positive definiteness of MPDATA is not affected by the compatibility issue [53]. The subsequent Section 4.2 reviews the NFT advection scheme MPDATA for the solution of (21). Section 4.2 is concerned with the MPDATA compatibility in regard to the GMCL (22). Section 4.4 investigates the issue of satisfying the GCL (24) in the NFT integration. Associated numerical scalar advection test experiments are provided in Section 5.

\subsection{Flux-form NFT advection scheme MPDATA}

Following [12], the FT discretisation of (21) underlying the derivation of MPDATA is

$$
\frac{\rho^{* n+1} \psi^{n+1}-\rho^{* n} \psi^{n}}{\delta \bar{t}}+\bar{\nabla} \cdot\left(\left(\rho^{*} \overline{\mathbf{v}}^{*}\right)^{n+1 / 2} \psi^{n}\right)=0
$$

the $\mathcal{O}(\delta \bar{t})$ truncation errors of which are delegated to corrective iterations of the scheme (highlighted below). As discussed in [54,13], the solution update for a time-dependent $\rho^{*}$, can be written as a solution update for the algorithm that assumes a time-independent $\rho^{*}$, multiplied by the ratio $\left(\rho^{* n} / \rho^{* n+1}\right)$. To see this, the semi-discretised Eq. (25) is recast into

$$
\psi^{n+1}=\frac{\rho^{* n}}{\rho^{* n+1}}\left[\psi^{n}-\frac{\delta \bar{t}}{\rho^{* n}} \bar{\nabla} \cdot\left(\left(\rho^{*} \overline{\mathbf{v}}^{*}\right)^{n+1 / 2} \psi^{n}\right)\right],
$$

where the expression in square brackets is formally independent of $\rho^{* n+1}$. A second-order accurate solution update under time-dependent coordinates can therefore be written as

$$
\psi_{\mathbf{i}}^{n+1}=\frac{\rho_{\mathbf{i}}^{* n}}{\rho_{\mathbf{i}}^{* n+1}} \widetilde{\mathcal{A}}_{\mathbf{i}}\left(\psi^{n},\left(\rho^{*} \overline{\mathbf{v}}^{*}\right)^{n+1 / 2}, \rho^{* n}\right)=\frac{\rho_{\mathbf{i}}^{* n}}{\rho_{\mathbf{i}}^{* n+1}} \psi_{\mathbf{i}}^{(I O R D)},
$$

in which $\widetilde{\mathcal{A}}$ denotes the MPDATA scheme for time-independent $\rho^{*}$; i.e. the same algorithm as used for time-independent, though curvilinear, coordinates. Specifically, $\widetilde{\mathcal{A}}$ iterates for $k=1, \operatorname{IORD}$ the discrete flux-form

$$
\psi_{\mathbf{i}}^{(k)}=\psi_{\mathbf{i}}^{(k-1)}-\frac{1}{\rho_{\mathbf{i}}^{* n}} \sum_{I=1}^{N}\left\{F\left(\psi_{\mathbf{i}}^{(k-1)}, \psi_{\mathbf{i}+\mathbf{e}_{I}}^{(k-1)}, V_{\mathbf{i}+1 / 2 \mathbf{e}_{I}}^{I(k)}\right)-F\left(\psi_{\mathbf{i}_{-} \mathbf{e}_{I}}^{(k-1)}, \psi_{\mathbf{i}}^{(k-1)}, V_{\mathbf{i}-1 / 2 \mathbf{e}_{I}}^{I(k)}\right)\right\},
$$

with $\mathbf{e}_{I}$ denoting the unit vector in the Ith of the $N$ spatial dimensions, while integer and half integer indices correspond to the cell centers and edges, respectively. The superscript in parentheses denotes the number of inner MPDATA iterations, not the time levels. The upwind flux functions $F$ in (28) can be stated in a symbolic form as

$$
F\left(\psi_{L}, \psi_{R}, V\right) \equiv 0.5\left((V+|V|) \psi_{L}+(V-|V|) \psi_{R}\right) .
$$

The algorithm is initialised with $\psi^{(0)} \equiv \psi^{n}, V^{I(1)} \equiv \bar{\lambda}^{I}\left(\rho^{*} \bar{v}^{* I}\right)^{n+1 / 2}$, and $\bar{\lambda}^{I} \equiv \delta \bar{t} / \delta \bar{x}^{I}$, where $\delta \bar{x}^{I}$ are spatial grid increments in the respective coordinate directions. Assumed here is the availability of an $\mathcal{O}\left(\delta \bar{t}^{2}\right)$ estimate for the generalised local Courant number $\bar{\lambda}^{I}\left(\rho^{*} \bar{v}^{* I}\right)^{n+1 / 2}$ at the intermediate time level $\bar{t}^{n+1 / 2}$ [12], see Appendix A for the particular computation in our model. The functional dependence of the error-compensative pseudo-velocities for the IORD $>1$ scheme can be written as

$$
V^{I(k)}=V^{I}\left(\mathbf{V}^{(k-1)}, \psi^{(k-1)}, \bar{\nabla} \psi^{(k-1)}, \rho^{* n}\right),
$$

where the appearance of $\psi$ in (30) indicates the fundamental nonlinear character of the scheme. The explicit expressions of the pseudo-velocities (30) underlying MPDATA can be found in [12].

\subsection{MPDATA compatibility with the GMCL}

A systematic analysis of the MPDATA scheme for the advective transport of a uniform scalar field $\psi_{c}$ under time-dependent generalised coordinates is conducted. The purpose is to investigate the compatibility of MPDATA for the solution of the scalar conservation law (21) with the GMCL (22).

\subsubsection{Analysis of the scheme}

Consider first the IORD = 1 variant of MPDATA, i.e. the first-order accurate, generic upwind scheme. Insertion of the uniform field $\psi^{n} \equiv \psi_{c} \neq 0$ in the MPDATA scheme (27)-(29) results in

$$
\psi_{\mathbf{i}}^{n+1}=\frac{\psi_{c}}{\rho_{\mathbf{i}}^{* n+1}}\left\{\rho_{\mathbf{i}}^{* n}-\sum_{I=1}^{N} \bar{\lambda}^{I}\left(\left(\rho^{*} \bar{v}^{* I}\right)_{\mathbf{i}+1 / 2 \mathbf{e}^{I}}^{n+1 / 2}-\left(\rho^{*} \bar{v}^{* I}\right)_{\mathbf{i}-1 / 2 \mathbf{e}^{l}}^{n+1 / 2}\right)\right\} .
$$


It shows that for constancy preservation of the generic upwind scheme (or, more generally, any linear FT scheme), it is necessary to assure that for a constant field the scheme reduces to

$$
1=\frac{1}{\rho_{\mathbf{i}}^{* n+1}}\left\{\rho_{\mathbf{i}}^{* n}-\sum_{I=1}^{\mathrm{N}} \bar{\lambda}^{I}\left(\left(\rho^{*} \bar{v}^{* I}\right)_{\mathbf{i}+1 / 2 \mathbf{e}^{l}}^{n+1 / 2}-\left(\rho^{*} \bar{v}^{* I}\right)_{\mathbf{i}-1 / 2 \mathbf{e}^{l}}^{n+1 / 2}\right)\right\} .
$$

Consequently, satisfying the discrete implementation of the GMCL (22)

$$
\rho_{\mathbf{i}}^{* n+1}=\rho_{\mathbf{i}}^{* n}-\sum_{I=1}^{\mathrm{N}} \bar{\lambda}^{I}\left(\left(\rho^{*} \bar{v}^{* I}\right)_{\mathbf{i}+1 / 2 \mathbf{e}^{l}}^{n+1 / 2}-\left(\rho^{*} \bar{v}^{* I}\right)_{\mathbf{i}-1 / 2 \mathbf{e}^{l}}^{n+1 / 2}\right)
$$

suffices for constancy preservation in (31). Yet, the MPDATA scheme (27) for IORD > 1 cannot be fully compatible with the GMCL, as (32) is already violated in the first iteration of (28)

$$
\psi_{\mathbf{i}}^{(1)}=\frac{\psi_{c}}{\rho_{\mathbf{i}}^{* n}}\left\{\rho_{\mathbf{i}}^{* n}-\sum_{I=1}^{\mathrm{N}} \bar{\lambda}^{I}\left(\left(\rho^{*} \bar{v}^{* I}\right)_{\mathbf{i}+1 / 2 \mathbf{e}^{I}}^{n+1 / 2}-\left(\rho^{*} \bar{v}^{* I}\right)_{\mathbf{i}-1 / 2 \mathbf{e}^{l}}^{n+1 / 2}\right)\right\},
$$

as opposed to (31). The field $\psi^{(1)}$ on the lhs of (34) differs locally by a factor of $\rho^{* n+1} / \rho^{* n}$ from the uniform field $\psi_{c}$, even if (33) is satisfied exactly. For the subsequent $k=2, \ldots, I O R D$ iterations, this non-uniform intermediate solution $\psi^{(1)}$ then generally yields non-zero pseudo-velocities $\mathbf{V}^{(k)} \sim\left(1 / \psi^{(k-1)}\right) \bar{\nabla} \psi^{(k-1)}$ according to (30).

\subsubsection{Extension of the scheme}

The previous discussion revealed that, given the validity of the discrete GMCL (33), the preservation of a uniform field $\psi_{c}$ requires compatibility of each MPDATA upwind iteration with (32). This can be achieved by using pseudo-velocity expressions that are isomorphic to the original expressions from the MPDATA literature [12] but with the value for the current iterate $\psi^{(k-1)}$ redefined as

$$
\hat{\psi}_{\mathbf{i}}^{(k-1)}:=\psi_{\mathbf{i}}^{(k-1)}\left(\frac{\rho_{\mathbf{i}}^{* n}}{\rho_{\mathbf{i}}^{* n+1}}\right),
$$

so that the pseudo-velocity functional form (30) becomes

$$
V^{I(k)}=V^{I}\left(\mathbf{V}^{(k-1)}, \hat{\psi}^{(k-1)}, \bar{\nabla} \hat{\psi}^{(k-1)}, \rho^{* n}\right) .
$$

The field $\hat{\psi}_{\mathbf{i}}^{(1)}$ that enters (36) for the first corrective step in the IORD $>1$ scheme already satisfies (31), and it remains uniform given the validity of (33). Consequently, the pseudo-velocity $\mathbf{V}^{(2)} \sim\left(1 / \hat{\psi}^{(1)}\right) \bar{\nabla} \hat{\psi}^{(1)}[12,13]$ is zero as a result. Therefore, there is no further contribution to the flux divergence in (28) for subsequent corrective iterations $k=2$, IORD, although the intermediate solution $\psi^{(1)}$ that enters $(28)$ is non-uniform. In summary, given the pseudo-velocities by the functional form (36) - instead of the original form (30) - and the validity of the discrete GMCL (33), the MPDATA scheme achieves exact preservation of a uniform advected field $\psi$, under arbitrary time-dependent generalised coordinates.

Noteworthy, both the original (30) and the redefined form of the pseudo-velocities (36) retain the second-order accuracy of the MPDATA scheme. From (33), the density-correction factor in (35) is $\rho^{* n} / \rho^{* n+1}=1+\mathcal{O}(\delta \bar{t})$. Because the error-compensative pseudo-velocities are $\mathcal{O}(\delta \bar{t})$ by design, the density-correction factor enters the $k>1$ iterations at $\mathcal{O}\left(\delta \bar{t}^{2}\right)$. The latter also indicates that the compatibility errors with the original form (30) occur at higher than second-order accuracy. Elementary numerical tests for the advection of a smooth scalar distribution under a prescribed oscillating mesh confirm the second-order asymptotic accuracy of the MPDATA advection for both forms of pseudo-velocities (Appendix B). A similar conclusion can be drawn for the stability properties; cf. Chapter 3.2 of [55].

Given that both forms of the pseudo-velocities result in the same overall accuracy of the scheme, and only one is fully compatible with the GMCL (22), the reader may wonder whether there is any advantage to the original scheme (27)(30). Insofar as mere scalar advection is concerned, the compatible form will be shown superior. However, there is a nonnegligible overhead when integrating the entire system $(2 a)-(2 c)$. Thus, the original form is still a viable option for slowly varying smooth meshes, or smooth fluid problems independent of the monotonicity of advection.

\subsection{Ensuring volume conservation for scalar advection under time-dependent coordinates}

In the theoretical discussion of Section 4.3, the discrete implementation (33) of the GMCL (22) has been postulated to be exactly satisfied. Here, we consider the solution of (21) assuming an externally specified solenoidal mass flux field $\rho^{*} \overline{\mathbf{v}}^{s}$ that satisfies the discrete anelastic mass continuity Eq. (13) consistent with the fluid model (2a)-(2c). Our concern is to assure the validity of the GCL (24) with a specified accuracy threshold.

In accordance with the discrete GMCL (33), the discrete representation of the GCL (24) is given as

$$
\rho_{\mathbf{i}}^{* n+1}=\rho_{\mathbf{i}}^{* n}-\sum_{I=1}^{\mathrm{N}} \bar{\lambda}^{I}\left(\left(\rho^{*} \bar{v}^{g I}\right)_{\mathbf{i}+1 / 2 \mathbf{e}^{l}}^{n+1 / 2}-\left(\rho^{*} \bar{v}^{g I}\right)_{\mathbf{i}-1 / 2 \mathbf{e}^{l}}^{n+1 / 2}\right) .
$$


The generalised density $\rho^{*}$ can be calculated from the coordinates of the mesh. Because the adaptive mesh is updated from $\bar{t}^{n}$ to $\bar{t}^{n+1}$ prior to evaluating the advective transport of auxiliary dependent variables, $\mathcal{A}_{\mathbf{i}}(\tilde{\psi})$ in $(9)$, both $\rho^{* n}$ and $\rho^{* n+1}$ enter $(37)$ as diagnostic quantities. The mesh mass flux $\left(\rho^{*} \bar{v}^{g I}\right)^{n+1 / 2}$ on the rhs of (37) is calculated from the coordinates of the adaptive moving mesh at different discrete time levels using a selected numerical approximation (to be discussed shortly). Therefore, all variables that enter (37) in the solution algorithm are predetermined and computed independently on the discrete mesh. As a consequence, the terms in (37) on the lhs are not necessarily equal to the terms of rhs in general, which can lead to significant errors in the advective transport (27).

The errors in the representation of the discrete GCL (37) depend strongly on the method of calculating the fluxes $\left(\rho^{*} \overline{\mathbf{v}}^{g}\right)^{n+1 / 2}$ at the local cell boundaries [27,29]. In Appendix A, a procedure for predicting the generalised contravariant mass flux $\left(\rho^{*} \overline{\mathbf{v}}^{*}\right)^{n+1 / 2}$ in (27) is given which minimises errors in the discrete representation of (37). Nonetheless, under general solution-adaptive moving meshes the discrete GCL (37) may still be in error. Therefore, a diagnostic approach is developed to ensure the validity of the discrete GCL (37) with sufficient accuracy in the integration.

Using the semi-discretised vectorial representation as in (25), the form of the GCL (24) consistent with the FT approximation in (25) becomes

$$
\frac{\left(\rho^{* n+1}-\rho^{* n}\right)}{\delta \bar{t}}+\bar{\nabla} \cdot\left(\rho^{*} \overline{\mathbf{v}}^{g}\right)^{n+1 / 2}=0 .
$$

For prescribed $\rho^{* n}$ and $\rho^{* n+1}$, a preliminary guess $\overline{\mathbf{v}}_{*}^{g}$ for the mesh velocity $\overline{\mathbf{v}}^{g}$ (Appendix A) is corrected to satisfy (38). For this purpose, a potential $\phi$ is introduced as

$$
\left\{\overline{\mathbf{v}}^{g}=\overline{\mathbf{v}}_{*}^{g}-\widetilde{\mathbf{G}}^{T} \widetilde{\mathbf{G}} \bar{\nabla} \phi\right\}_{\mathbf{i}}^{n+1 / 2} .
$$

Eq. (39) is derived as follows. Starting from the ansatz $\mathbf{v}^{g}=\mathbf{v}_{*}^{g}+\widetilde{\mathbf{G}} \bar{\nabla} \phi$ for the mesh velocity $\mathbf{v}^{g}=\partial \mathbf{x} / \partial \bar{t}$ in physical space $\mathbf{S}_{p}$, (39) results by insertion into the transformation relations $\overline{\mathbf{v}}^{g}=-\widetilde{\mathbf{G}}^{T} \mathbf{v}^{g}$ defined by the Kronecker delta (5). Ultimately, inserting (39) into (38) leads to an elliptic boundary value problem for $\phi$ of the form

$$
\left\{-\frac{\delta \bar{t}}{\rho^{* n+1 / 2}}\left(\frac{\left(\rho^{* n+1}-\rho^{* n}\right)}{\delta \bar{t}}+\bar{\nabla} \cdot\left(\rho^{*}\left[\overline{\mathbf{v}}_{*}^{g}-\widetilde{\mathbf{G}}^{T} \widetilde{\mathbf{G}} \bar{\nabla} \phi\right]\right)^{n+1 / 2}\right)\right\}_{\mathbf{i}}=0 .
$$

Again, multiplication by the factor $\left(-\delta \bar{t} / \rho^{*}\right)$ has been applied in (40) for numerical reasons, cf. the statements following (13) in Section 2.2. The formulated elliptic problem (40) is solved subject to either periodic or Dirichlet boundary conditions for the mesh velocity $\overline{\mathbf{v}}^{g}$ at the intermediate time level $\bar{t}^{n+1 / 2}$. The Dirichlet boundary conditions along $\partial \mathcal{D}_{t}$ prescribed on the normal component of the mesh velocity $\overline{\mathbf{v}}^{g} \cdot \overline{\mathbf{n}}$, which are subject to the integrability condition $\int_{\mathcal{D}_{t}} \partial \rho^{*} / \partial \bar{t} d \bar{V}+$ $\int_{\partial \mathcal{D}_{t}} \rho^{*} \overline{\mathbf{v}}^{g} \cdot \overline{\mathbf{n}} d \bar{\sigma}=0$, imply the correct Neumann boundary conditions for $\phi$. The metric coefficients contained in $\widetilde{\mathbf{G}}$ and the generalised density $\rho^{*}$ at $\bar{t}^{n+1 / 2}$ are straightforwardly obtained to second-order accuracy, see Appendix A. The GCR iterative solver (see Section 2.2) is applied to solve (40). The stopping criterion for the iterative solver is based on

$$
\left\|r_{g c l}\right\|_{\infty}=\left\|\frac{\delta \bar{t}}{\rho^{* n+1 / 2}}\left(\frac{\left(\rho^{* n+1}-\rho^{* n}\right)}{\delta \bar{t}}+\bar{\nabla} \cdot\left(\rho^{*} \overline{\mathbf{v}}^{g}\right)^{n+1 / 2}\right)\right\|_{\infty}<\epsilon .
$$

The proposed diagnostic approach differs from the common prognostic approach for the GCL [25,29,56]. The latter approach employs the GCL (24) as an auxiliary prognostic equation for $\rho^{*}$. Specifically, in the framework of our FT solver, it uses the discrete approximation (37). In anelastic systems, the prognostic treatment of the GCL (37) leads to an over-specified mathematical problem as $\rho^{*}$ is a known diagnostic variable. In contrast, the diagnostic approach presented above corrects the temporal approximation of the mesh velocity $\overline{\mathbf{v}}^{g}$ as derived from the mesh coordinates $\mathbf{x}^{n}$ and $\mathbf{x}^{n+1}$ (Appendix A). However, the prognostic approach is appealing as it is simple, direct, and satisfies the GCL (37) to machine precision. For a comparison with the diagnostic approach, the prognostic approach for the GCL (37) is algorithmically implemented as follows. At the initial time of the integration, $\rho_{\mathbf{i}}^{* n} \equiv \rho_{\mathbf{i}}^{* 0}$ in (37) is diagnostically computed from the mesh. Henceforth, $\rho_{\mathbf{i}}^{* n}$ is updated with the prognostically computed value $\rho_{i}^{* n+1}$ from (37) - which is in the manner as described for instance by [29]. The fluxes $\left(\rho^{*} \overline{\mathbf{v}}^{g}\right)^{n+1 / 2}$ on the rhs of (37) are always diagnostically derived using the procedure given in Appendix A. In Section 5, it is demonstrated that the predicted generalised density $\rho^{*}$ in the prognostic approach can depart significantly from its diagnostic counterpart as the integration proceeds, thereby introducing errors in the advective scalar transport.

\section{Scalar advection experiments}

Scalar advection experiments solving (21) with $\rho_{b} \equiv 1\left(\rho^{*} \equiv \bar{G}\right)$ in two spatial dimensions are used to investigate the developed framework of the solution-adaptive moving mesh solver. The various implementations of the integration scheme discussed in the previous sections are compared, and their relative merits are assessed.

The adopted experimental setup follows [57]: A scalar variable $\psi$, with an initial cosine hill profile, transforms into a filamentary structure in the response to a prescribed, variable (in time and space) deformational flow field. The flow field, defined as a perturbation to a background solid-body rotation, reverses its magnitude in time to recover the initial condition after multiples of $T / 2$, where $T=1.0$ defines one period. Thus, the test problem allows straightforward evaluation of the 
numerical results due to the availability of the analytical solution at $T / 2, T, 3 T / 2, \ldots$. The detailed specifications including analytic formulae for the initial scalar distribution $\psi$ and the stream function $\Psi$ (defining the flow field) are given in [57]; Eqs. (39) and (A.6) therein, respectively. The lengths of the model domain $\mathcal{D}_{p}$ are $L_{x}=L_{y}=1.0$. Here, a non-zero background value of $\psi_{0}=0.5$ for the initial scalar distribution $\psi$ according to the formula (39) in [57] is used. Moreover, in the generalised coordinate formulation considered here, the components of the solenoidal velocity $\overline{\mathbf{v}}^{s}$ in $\mathbf{S}_{t}$ are evaluated on the discrete grid according to $\overline{\mathbf{v}}^{s}=\rho^{*-1} \overline{\mathbf{k}} \times \bar{\nabla} \Psi$, where $\overline{\mathbf{k}}$ is the vertical unit vector. Importantly, this procedure ensures to satisfy the divergence constraint $(2 \mathrm{c})$ to machine precision throughout the integration.

The solution of (21) is coupled with the MMPDE apparatus described in Section 3. The monitor function (18) employs the refinement indicator $\Phi\left(t, \mathbf{x}_{h}\right)=\left\|\nabla_{h} \psi\left(t, \mathbf{x}_{h}\right)\right\|$ in the weighting function (19), where $\|\cdot\|$ refers to the Euclidean vector norm. The use of this refinement criterion is motivated by the heuristic argumentation that relatively large solution errors in the advection scheme arise in regions of greatest $\psi$ variations. The parameter $\beta$ in (19) is set to $\beta=0.5$, which means that approximately half of the mesh points are placed in the refinement regions where the weighting function (19) is large [10,51]. The mesh relaxation time is set to $\mathcal{T}=1.6 \cdot 10^{-2}$. This value is roughly 20 times the average time step $7 \cdot 10^{-4}$.

The third-order accurate (for constant coefficients) version [12] of MPDATA with non-oscillatory enhancement [21] is applied. The third-order scheme minimises the solution dependence on local Courant numbers, and thus benefits calculations on highly variable meshes. On the other hand, it well illustrates theoretical considerations of Section 4 , as the compatibility issue manifests itself similarly in the second-order accurate version of MPDATA. All simulations are performed using variable time stepping with a fixed maximum Courant number $\mathcal{C}_{\max }=0.5$. For comparison, the corresponding mesh-movement Courant number, based on the velocity $\overline{\mathbf{v}}^{g}$, is roughly 0.2 with standard deviation 0.14 . Table 1 summarises the various implementations of the NFT integration that are applied in the experiments. The following discussion presents first the results obtained with the particular implementation RD developed in Sections 4.3 and 4.4. By the end of this section, the scheme $\mathrm{RD}$ is compared against the other schemes given in Table 1.

Fig. 1 shows the scalar field $\psi$ overlaid by the adaptive moving mesh at the initial time $t=0$ and after $t=0.25 T$ of the model integration. The mesh at $t=0$ already provides high resolution in the region where values of $\|\nabla \psi\|$ are relatively large. This is achieved by reapplying the MMPDE integration procedure to the initial distribution of the scalar $\psi$ until the desired degree of adaptation is attained (typically until a steady state of the mesh movement). At $t=0.25 T$, the circularly-symmetric zone of nonzero $\psi$ values from $t=0$ is stretched into a thin elongated structure. Large local gradients of $\psi$ exist transverse to the elongation direction, and the adaptive solver reliably provides a finer mesh there. This locally fine mesh is essential to resolve the large gradients in the transported field $\psi$. The remaining parts of the domain are more coarsely resolved. The ratio of the smallest to the largest mesh cell area at $t=0.25 T$ is $1 / 24$.

Fig. 2 illustrates the efficacy of the simulations. The final result at $t=T$ of the adaptive solver is compared to results obtained using a non-adaptive static uniform mesh. The results show that the $L_{2}$ error norm (rms error) and the $L_{\infty}$ error norm for the solution-adaptive mesh run in Fig. $2 \mathrm{c}$ are about one order of magnitude lower than for the static mesh run in Fig. 2a, even though both runs use the same number of mesh cells $\mathrm{N}_{c}=50^{2}$. Fig. $2 \mathrm{c}$ documents that the adaptive solution accurately reproduces the amplitude, position, and shape of the cosine hill in the advected field. When compared to another static uniform mesh run with a total of $\mathrm{N}_{c}=250^{2}$ mesh cells, the adaptive run with $\mathrm{N}_{c}=50^{2}$ still shows, respectively, $\sim 1.1$ and $\sim 1.4$ times lower $L_{2}$ and $L_{\infty}$ error norms.

The relative wall clock time of the three different simulations is given by the variable $\mathrm{T}_{\mathrm{rw}}$ in Fig. 2 . The adaptive simulation in Fig. $2 c$ requires a factor of 5.5 larger wall clock time $T_{w}$ than the uniform mesh simulation with the same number of mesh cells in Fig. 2a. The computational overhead of the adaptive simulation over the uniform mesh simulation in Fig. 2a results from the use of smaller time steps with $\mathcal{C}_{\max }=0.5$ due to the higher spatial resolution, plus the processing of the MMPDE apparatus. However, the adaptive mesh simulation in Fig. $2 \mathrm{c}$ requires a factor of 24 less wall clock time $\mathrm{T}_{\mathrm{w}}$ than the highresolution uniform mesh run in Fig. 2b. Altogether, this demonstrates the high efficacy of the solution-adaptive moving mesh solver for the present configuration. The $L_{2}$ and $L_{\infty}$ error norms of Fig. 2 can also be compared to the results given in Blossey and Durran [57], Section 4.2 therein. Using a static uniform mesh of $\mathrm{N}_{c}=200^{2}$, values of $L_{2} / L_{\infty}=0.009 / 0.132$ or $L_{2} / L_{\infty}=0.003 /$

\section{Table 1}

Summary of the various implementations of the NFT integration compared in the numerical experiments. The first column designates the implementation. The second column states whether the pseudo-velocities in MPDATA use the original form (30) (denoted as 0 ) or the densitycorrection factor (35) in the redefined form (36) (denoted as R). The third column describes the formulation of the discrete GCL: either the use of the standard (uncorrected) algorithm (47)-(49) (denoted as S), the diagnostic approach applying a correction to the mesh velocity $\overline{\mathbf{v}}^{g}$, i.e. treatment of the GCL as the elliptic problem (40) (denoted as D), or the prognostic approach for the GCL (37) (denoted as PP; for consistency with the presentation in [55]). See Sections 4.2, 4.3 and 4.4 for the explanation of the various schemes.

\begin{tabular}{lll}
\hline Implementation & Density-correction & GCL formulation \\
\hline OS & No & standard \\
RS & Yes & standard \\
RPP & Yes & prognostic \\
RD & Yes & diagnostic \\
\hline
\end{tabular}



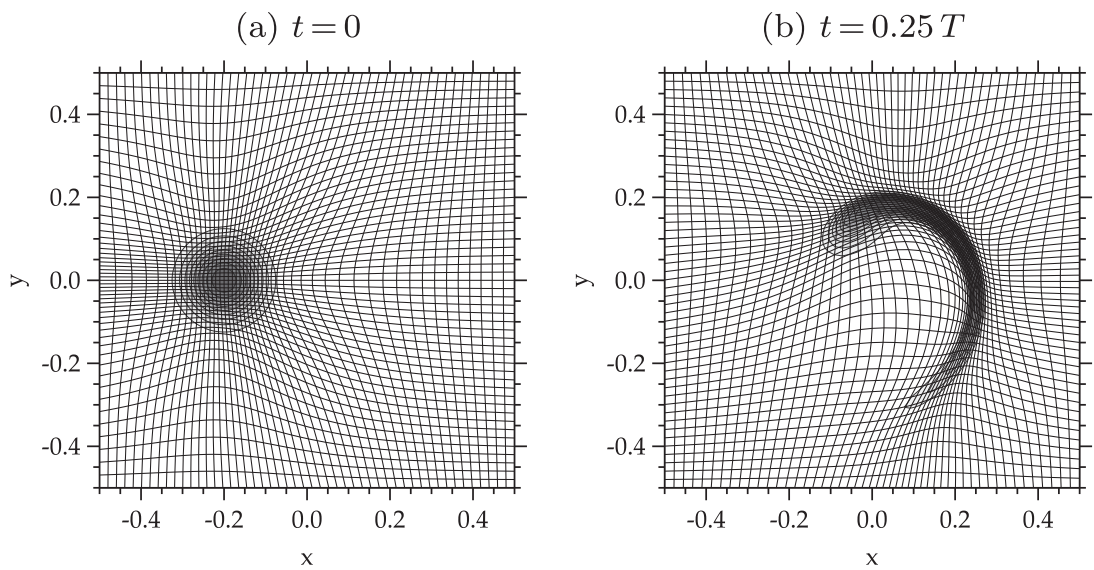

Fig. 1. MPDATA integration of the scalar advection problem with a solution-adaptive mesh at the initial time (a), and at $0.25 T$ (b). Indicated are contours of the transported scalar variable $\psi$ (shaded; darker shading corresponds to larger value of $\psi$ ), overlaid by the solution-adaptive mesh (black solid lines).

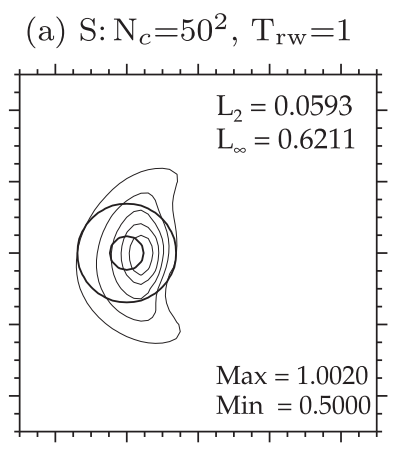

(b) $\mathrm{S}: \mathrm{N}_{c}=250^{2}, \mathrm{~T}_{\mathrm{rw}}=133$

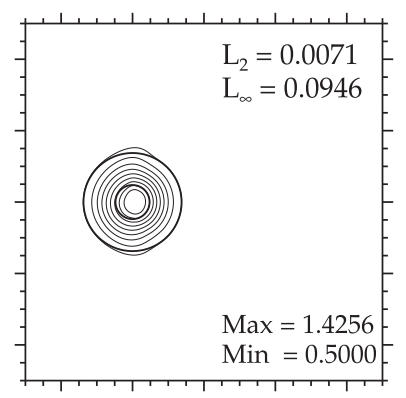

(c) A: $\mathrm{N}_{c}=50^{2}, \mathrm{~T}_{\mathrm{rw}}=5.5$

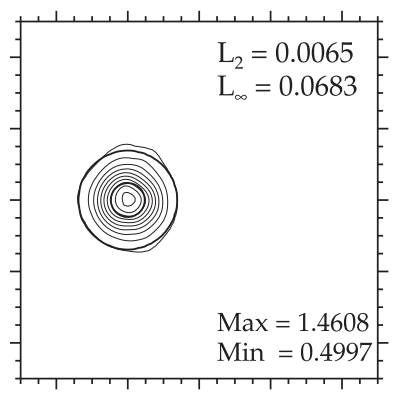

Fig. 2. Comparison of results from the integration of the scalar advection problem with a static uniform mesh (a,b), against the integration that uses solution-adaptive meshing (c). Shown are contours of the numerical solution for the scalar field $\psi$ at the final integration time $t=T$ (thin solid lines; contours from 0.55 to 1.45 at intervals of 0.1 ). The analytical solution is overlaid (heavy solid lines; shown are only the contour values of 0.55 and 1.25 ). Domain maximum and minimum values of the numerical solution for $\psi$, plus standard $L_{2}$ and $L_{\infty}$ error norms, are given in the lower and upper right parts of the figure, respectively. The symbol $\mathrm{N}_{c}$ refers to the number of discrete mesh cells, and $\mathrm{T}_{\mathrm{rw}}$ is the relative wall clock time with respect to the low-resolution static uniform mesh run that uses $\mathrm{N}_{c}=50^{2}$ in (a).

0.050 were obtained employing a piecewise parabolic method (PPM) with global monotonicity preservation or an unsplit weighted essentially non-oscillatory method (WENO), respectively.

The results presented in Figs. 1 and 2 are obtained with the particular scheme RD (Table 1) that combines the redefined pseudo-velocities (36) in MPDATA with the diagnostic approach for the treatment of the GCL (24). Here, the elliptic problem for the GCL (40) is solved with the residual error defined by (41) to be less than $\epsilon=10^{-5}$. With this threshold $\epsilon$, the applied GCR solver required an average number of merely 4 iterations to converge.

Fig. 3 compares the solution quality of the proposed scheme RD against the other schemes OS, RS, and RPP listed in Table 1 . The $L_{2}$ and $L_{\infty}$ error norms obtained with the schemes OS, RS, and RPP are considerably larger than with the scheme RD. For instance, the $L_{2}$ and $L_{\infty}$ error norms for OS are $\sim 2.4$ and $\sim 2$ times larger than for RD, respectively. In addition, the schemes OS and RS suffer from a significant loss of the solution monotonicity. Spurious minima (see the dashed contour lines) exist with OS in Fig. 3a that undershoot the analytical minimum of 0.5 at about $6 \%$. The scheme OS also creates values of $\psi$ that overshoot the analytical maximum of 1.5 . The solution with RD shows a minimum of $\psi=0.4997$, which is $0.06 \%$ below the analytical minimum. Also, the solution quality is improved by using RS instead of OS.

The scheme RPP provides a solution that is completely free of spurious extrema. The $L_{2}$ and $L_{\infty}$ error norms for RPP are nonetheless $\sim 1.8$ and $\sim 2$ times larger than for RD (Fig. 3). An investigation in the source of the considerably larger errors with RPP versus RD was conducted. It revealed that, as the integration advances in time, the prognostically computed generalised density $\rho^{*}$ by means of the GCL (37) deviates significantly from the diagnostically computed $\rho^{*}$ determined from the actual mesh (not shown). Thus, errors in the numerical approximation to the GCL (37) accumulate in the predicted $\rho^{*}$ with time, and this deteriorates the solution quality of $\psi$ in the advection scheme MPDATA. Substantiation for this finding is provided as follows. The scheme RPP can be modified in that $\rho^{*}$ is not treated as a purely prognostic variable but is reinitialised with its diagnostic value at every time step. Using this implementation, the prognostically computed $\rho^{*}$ is forced to remain 
(a) OS, $\mathrm{T}_{\mathrm{rw}}=1.0$

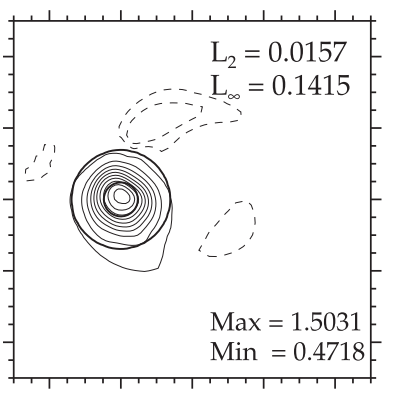

(b) RS, $\mathrm{T}_{\mathrm{rw}}=1.01$

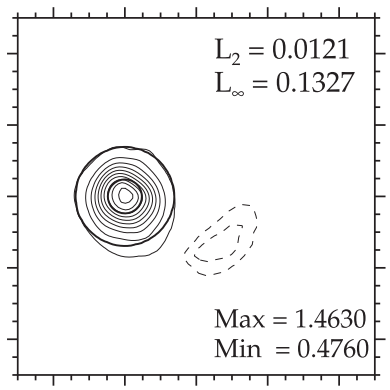

(d) RD, $\mathrm{T}_{\mathrm{rw}}=1.18$

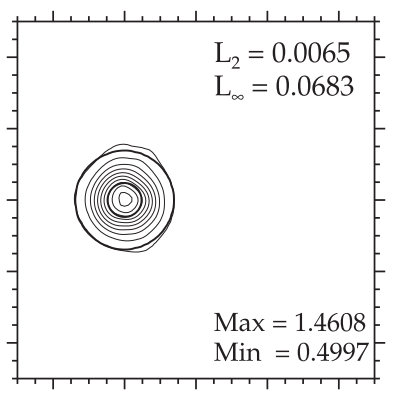

(c) RPP, $\mathrm{T}_{\mathrm{rw}}=1.04$

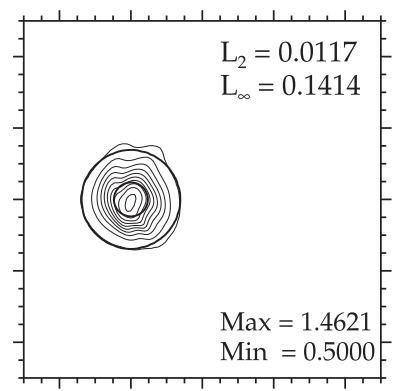

Fig. 3. As in Fig. 2c, but for the various implementations of the NFT integration listed in Table 1: (a) OS, (b) RS, (c) RPP, and (d) RD. Contours of $\psi$ lower than the initial minimum value of 0.5 are shown (dashed lines at intervals of -0.01 starting at 0.49 ). Here, $T_{\mathrm{rw}}$ is the relative wall clock time with respect to the original scheme OS.

close to its diagnostic counterpart, while still providing full compatibility with the GCL (37) as in RPP. The resulting error norms with this particular implementation are then $L_{2}=0.0067$ and $L_{\infty}=0.0829$, which is at order of the error norms obtained with RD. Note, the reinitialisation of the prognostic $\rho^{*}$ at every time step leads to the loss of the machine-precision conservation for the flux-form scalar advection scheme, which is why we consider the implementation not to be a viable alternative.

It is further revealing to investigate the validity of the GCL (24) in the discrete model by the various schemes. Using (41) as the accuracy measure, the schemes OS and RS both have maximum values of $\left\|r_{g c l}\right\|_{\infty} \sim 7.0 \cdot 10^{-4}$ during the simulation. The scheme RS can directly be compared to the scheme RD, as these two schemes employ the identical form of MPDATA. The more accurate representation of the GCL to $\left\|r_{g c l}\right\|_{\infty}=1.0 \cdot 10^{-5}$ using the diagnostic approach in the scheme RD results in the considerably more accurate MPDATA solution (Fig. 3). At this point, it is interesting to remark that the possible combination of a scheme OD does not yield a quality of the solution comparable to RD, but a solution quality similar to OS and RS (not shown). The latter confirms the requirement for the incorporation of the density-correction factor (35) in the pseudovelocities (36) of MPDATA under solution-adaptive moving meshes. Finally, the computational overhead of $14-18 \%$ (see the quantity $\mathrm{T}_{\mathrm{rw}}$ in Fig. 3) introduced by the solution of the elliptic problem for the GCL (40) in the diagnostic approach can be acceptable for many applications considering the improved solution quality with the scheme RD over the other schemes.

\section{Life cycle of a baroclinic wave instability}

The developed flow solver is applied to simulate multiscale interactions in an archetypal atmospheric flow problem. Baroclinic wave life cycle experiments are conducted that reflect the evolution of synoptic-scale weather systems (cyclones and anticyclones) in the mid-latitudes. The model problem is a dry inviscid baroclinic instability of an initially straight zonal jet flow in a periodic channel on the $f$-plane. Numerous studies used a similar problem to investigate the dynamics of fronts [58], the formation of tropopause folds [59], and the spontaneous generation and the propagation of internal gravity waves $[60,61]$. Recently, this model problem was also used to investigate the flow representation of distinct analytical forms of atmospheric sound-proof equations [62]. Furthermore, simulation of baroclinic instability underlies established benchmarks assessing the performance of global climate and weather models $[63,64]$. So far, the solvers employed static uniform meshes or prescribed grid nesting in certain regions. To the authors knowledge, the performance of adaptive moving mesh methods was not reported for this archetypal atmospheric flow problem. 


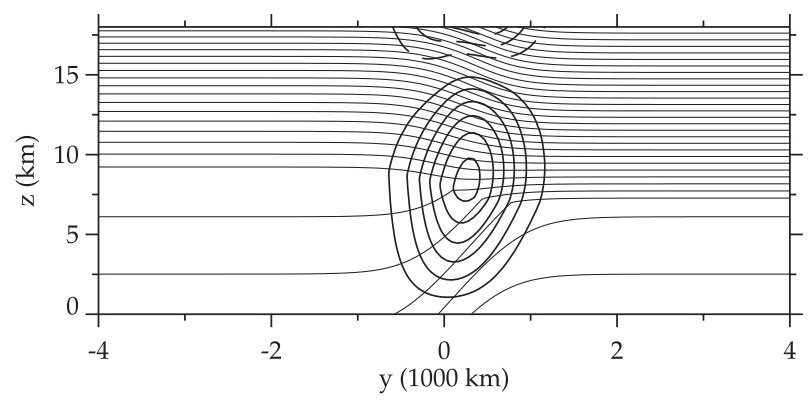

Fig. 4. Vertical $y-z$ cross section of the geostrophically balanced, baroclinically unstable ambient state for potential temperature $\theta_{e}$ (thin solid contour lines, $\Delta \theta_{e}=10 \mathrm{~K}$ ) and velocity $u_{e}$ (thick solid contour lines positive, thick dashed contour lines negative, $\Delta u_{e}=10 \mathrm{~m} \mathrm{~s}{ }^{-1}$, zero contour line not shown).

\subsection{Experimental design}

The simulation domain $\mathcal{D}_{p}$ defines a straight channel of length $L_{x}=10000 \mathrm{~km}$ that is periodic in the zonal direction $x$. The meridional width of the channel is $L_{y}=8000 \mathrm{~km}$, and its depth is set to $H=18 \mathrm{~km}$. Rigid free-slip boundaries confine the channel in $y$ and $z$. The variables in the anelastic Eqs. (2) are defined as follows. The gravitational acceleration is specified as $g=9.81 \mathrm{~m} \mathrm{~s}^{-2}$, and the components of the Coriolis parameter are set to $f_{3}=f=10^{-4} \mathrm{~s}^{-1}$ and $f_{1}=f_{2}=0$. Waveabsorbing devices near the top of the model domain are applied. The parameters $\alpha_{M}$ and $\alpha_{H}$, appearing in (2a) and (2b), increase linearly from a value of zero at $2 \mathrm{~km}$ below the top boundary to $600^{-1} \mathrm{~s}^{-1}$ at the boundary. The hydrostatic basic state profiles of density $\rho_{b}$ and potential temperature $\theta_{b}$ in the system (2) are defined using a constant stability parameter $S=N^{2} / g=1.2 \cdot 10^{-5} \mathrm{~m}^{-1}$ (where $N$ is the Brunt-Väisälä frequency) and the boundary values, at $z=0$, $\rho_{b}(0)=1.189 \mathrm{~kg} \mathrm{~m}^{-3}$ and $\theta_{b}(0)=285 \mathrm{~K}$. An ideal gas with constant specific heat capacities $c_{p}=1004 \mathrm{~J} \mathrm{~kg}^{-1} \mathrm{~K}^{-1}$ and $c_{v}=717 \mathrm{~J} \mathrm{~kg}^{-1} \mathrm{~K}^{-1}$ is assumed.

Here, the ambient state variables $\theta_{e}$ and $\mathbf{v}_{e}$ in the system (2) describe a geostrophically balanced but baroclinically unstable straight zonal jet. A zonally uniform potential temperature field $\theta_{e}(y, z)$ is prescribed following [59], see Appendix C. The resulting distribution of $\theta_{e}(y, z)$ is depicted in Fig. 4. A strong baroclinic zone exists in the centre of the channel. Given the prescribed field $\theta_{e}(y, z)$, the inertial flow field $\mathbf{v}_{e}=\left(u_{e}, 0,0\right)^{T}$ is obtained from the thermal wind relation

$$
\frac{\partial u_{e}}{\partial \bar{z}}=-\frac{g}{\theta_{b} f}\left(\widetilde{G}_{2}^{1} \frac{\partial \theta_{e}}{\partial \bar{x}}+\widetilde{G}_{2}^{2} \frac{\partial \theta_{e}}{\partial \bar{y}}\right)
$$

The relation (42) is derived from the anelastic Eqs. (2) assuming the identity transformation $\bar{z} \equiv z$. The flow field $u_{e}$ at $\bar{z}=0$ is defined to be zero. Then, numerical integration of (42) in the vertical direction yields $u_{e}(y, z)$, as shown in Fig. 4 . The resulting maximum velocity in the core of the computed jet flow is $\approx 63 \mathrm{~m} \mathrm{~s}^{-1}$ at the height of $z \approx 9 \mathrm{~km}$. To stimulate the baroclinic growth, the undisturbed ambient state of Fig. 4 is perturbed at the initial time $t=0$ by means of a thermal tropopause anomaly: $\theta^{\prime}(0, x, y, z)=\delta \theta \cos ^{2}(0.5 \pi \tilde{r}) \quad$ if $\quad \tilde{r} \leqslant 1$ (and 0 otherwise), where $\tilde{r}=\left([(x-5000 \mathrm{~km}) / 500 \mathrm{~km}]^{2}+[y / 500 \mathrm{~km}]^{2}+\right.$ $\left.[(z-9 \mathrm{~km}) / 2 \mathrm{~km}]^{2}\right)^{1 / 2}$, and $\delta \theta=3 \mathrm{~K}$. The initial flow field $\mathbf{v}$ is set equal to the ambient flow field $\mathbf{v}_{e}$.

The evolution of the baroclinic wave instability is simulated over a period of $T=288 \mathrm{~h}$. A compatible flux-form MPDATA advective transport with the GMCL (22) employs the implementation RD of Table 1 . The convergence threshold in the solution of the elliptic problems for the anelastic mass continuity (13) and the GCL (40) is set to $\epsilon=10^{-6}$. As the mesh adaptation by the MMPDE apparatus is independent of height (cf. Section 3), only a two-dimensional (horizontal) problem for the GCL (40) is solved. The mesh relaxation time in the MMPDE apparatus is set to $\mathcal{T}=2.0 \cdot 10^{5} \mathrm{~s}(\approx 55 \mathrm{~h})$. The selected time scale $\mathcal{T}$ is at the order of the reciprocal of the instability growth rate in the baroclinic wave evolution; however, in the range $10 \mathrm{~h}$ $<\mathcal{T}<60 \mathrm{~h}$, the simulated baroclinic instability is essentially insensitive to the choice of $\mathcal{T}$.

\subsection{Large-scale flow evolution}

The key question for the adaptive simulations of the 3D baroclinic flow evolution is how to specify the monitor function (18). A mesh refinement indicator $\Phi$ in (19) that has been found to work reliably is the vertical average of the horizontal potential temperature gradient (in the Euclidean norm) given as

$$
\Phi(t, x, y)=\frac{1}{H} \int_{0}^{H}\left\|\nabla_{h} \theta(t, x, y, z)\right\| d z .
$$

The use of (43) aims at an increased resolution of thermal frontal zones characteristic of the baroclinic flow. The average over the depth $H$ of the domain $\mathcal{D}_{p}$ in (43) accounts for the vertical variation of $\left\|\nabla_{h} \theta\right\|$ with height and combines the information in the horizontal monitor function $M\left(t, \mathbf{x}_{h}\right)$. Unless otherwise stated, all presented adaptive simulations use (43). Other specifications than (43) for the mesh refinement indicator $\Phi\left(t, \mathbf{x}_{h}\right)$ are investigated in the next section. The parameter $\beta$ in (19) is 
Table 2

Specifications of the simulations for the large-scale flow investigation. The first column designates each simulation. The symbols $\mathrm{S}$ and A indicate, respectively, the use of a static uniform mesh or a solution-adaptive moving mesh; whereas the numbers past $\mathrm{S}$ or A indicate the number of horizontal mesh cells employed. The second column specifies the number of mesh points in each coordinate direction $\mathrm{N}_{x} \times \mathrm{N}_{y} \times \mathrm{N}_{z}$. The third column lists spatial grid increments $\delta \bar{x} \times \delta \bar{y}$ in the horizontal (the vertical grid increment is $\delta \bar{z}=300 \mathrm{~m}$ in all simulations); whereas, the fourth column lists temporal increments $\delta \bar{t}$, selected to give a maximum value of $\mathcal{C}_{\max } \approx 0.5$. The last column provides the total wall clock time T $\mathrm{w}$ required for each simulation on 20 processors ( 21 for A6254) of an IBM p575 "Power6" cluster.

\begin{tabular}{llll}
\hline Simulation & $\mathrm{N}_{x} \times \mathrm{N}_{y} \times \mathrm{N}_{z}$ & $\delta \bar{x} \times \delta \bar{y}$ & $\delta \bar{t}$ \\
\hline S7050 & $95 \times 76 \times 61$ & $106 \mathrm{~km} \times 106 \mathrm{~km}$ & $360 \mathrm{~s}$ \\
S15429 & $140 \times 112 \times 61$ & $72 \mathrm{~km} \times 72 \mathrm{~km}$ & $240 \mathrm{~s}$ \\
S62217 & $280 \times 224 \times 61$ & $36 \mathrm{~km} \times 36 \mathrm{~km}$ & $120 \mathrm{~s}$ \\
A6254 & $119 \times 54 \times 61$ & $85 \mathrm{~km} \times 151 \mathrm{~km}$ & $150 \mathrm{~s}$ \\
\hline
\end{tabular}

set to $\beta=0.4$, i.e. about $40 \%$ of the available mesh points are placed in the refinement regions [51]. The adaptive simulations start from an optimised mesh with respect to the monitor function $M$ by applying the MMPDE apparatus at $t=0$.

An analytic solution is not available for the present flow problem. Therefore, the computed results from the solutionadaptive mesh simulations are validated against the results from reference simulations using a static uniform mesh. Specifications for the large-scale flow study are summarised in Table 2. With the selected MMPDE parameters in the adaptive simulations, the resulting minimum (maximum) mesh increments in physical space are $\sim 60 \mathrm{~km}(100 \mathrm{~km})$ in $\bar{x}$ and $\sim$ $60 \mathrm{~km}(270 \mathrm{~km})$ in $\bar{y}$, respectively.

After approximately 6 days of model integration a synoptic-scale baroclinic wave disturbance develops, and its amplitude grows at an exponential rate [59]. The wave growth saturates after around $t=240 \mathrm{~h}$ of model integration. Fig. 5 contrasts the adaptive simulation A6254 against the reference simulations S7050, S15429, and S62217 at $t=234 \mathrm{~h}$. The overall distribution of the $\theta$ field at $z=2 \mathrm{~km}$ altitude is qualitatively similar for the various simulations. The amplitude of the baroclinic wave and the temperature gradients of the frontal zones, however, are both significantly larger for higher resolutions. The adaptive simulation A6254 provides a solution comparable with the reference simulation S15429.

The performance of the adaptive solver is evaluated using statistical measures of kinetic energetics. Analysed are time series of total kinetic energy $\langle\mathrm{KE}\rangle$, zonal kinetic energy $\langle\mathrm{ZKE}\rangle$, and eddy kinetic energy $\langle\mathrm{EKE}\rangle$, where \langle\rangle denotes the spatial

(a) $\mathrm{S} 7050$

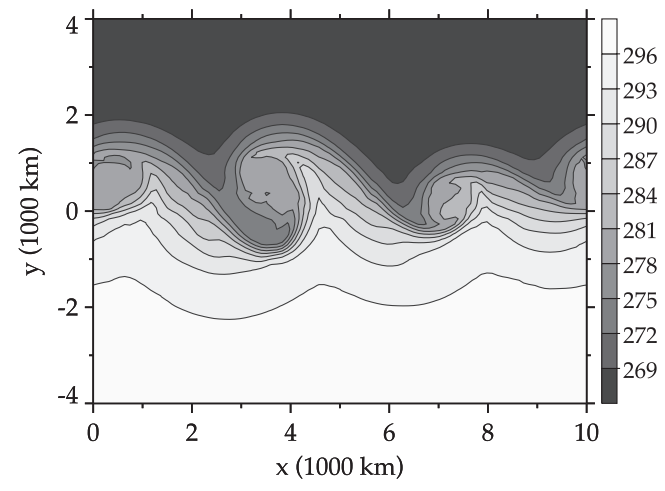

(c) $\mathrm{S} 62217$

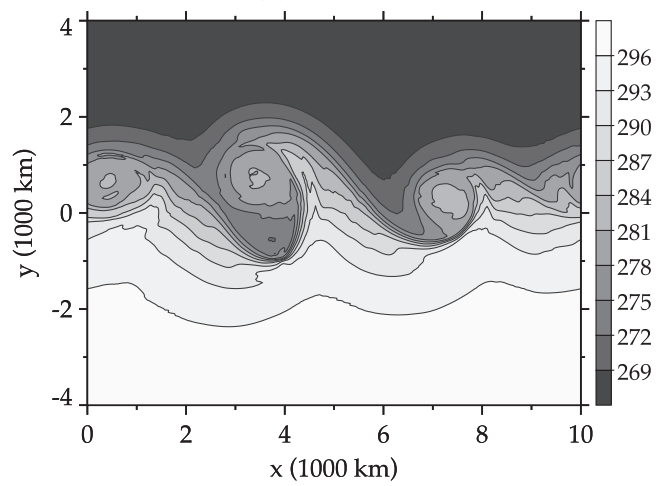

(b) S15429

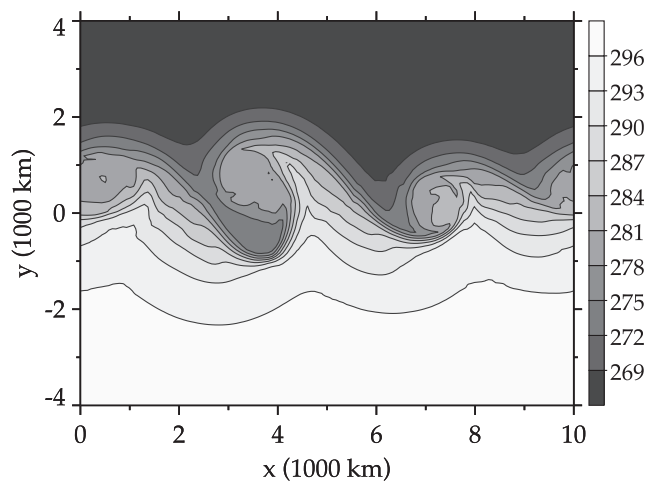

(d) A6254

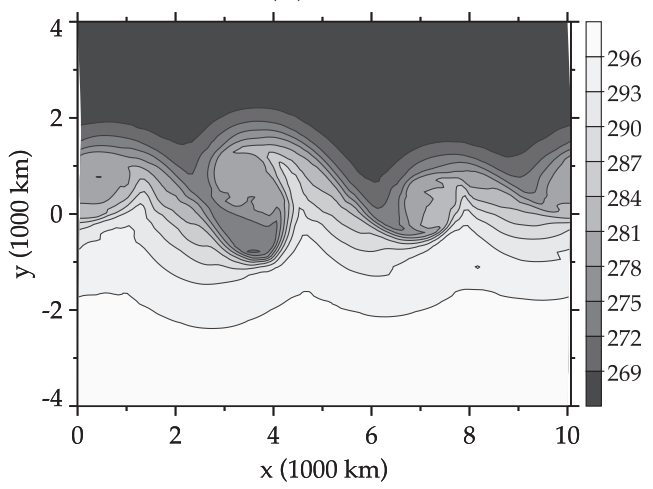

Fig. 5. Horizontal cross section of the potential temperature field $\theta$ (K, shaded and grey line contours) at the height $z=2 \mathrm{~km}$ after $t=234 \mathrm{~h}$ of simulation time. Comparison of the different simulations (a) S7050, (b) S15429, and (c) S62217, and (d) A6254 given in Table 2. 
(a) $\langle\mathrm{KE}\rangle$

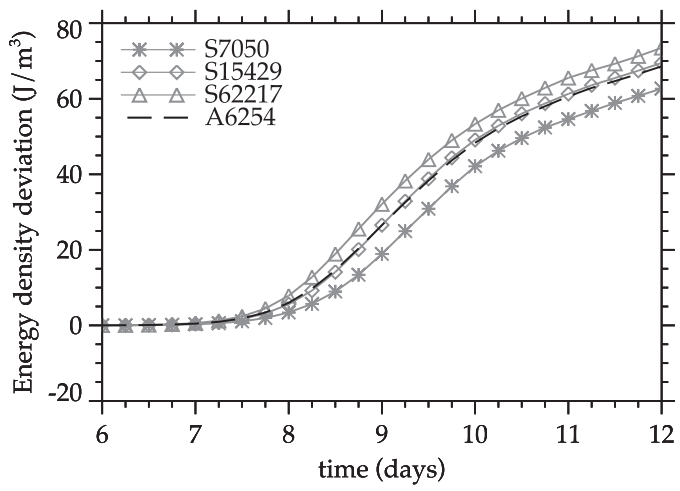

(b) $\langle\mathrm{ZKE}\rangle$

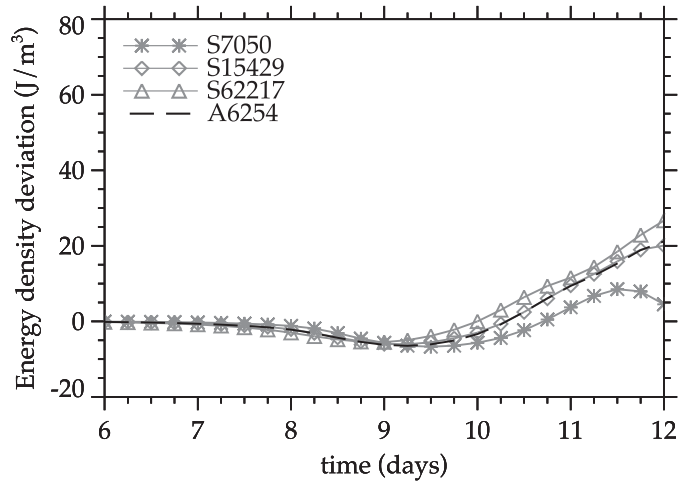

(c) $\langle\mathrm{EKE}\rangle$

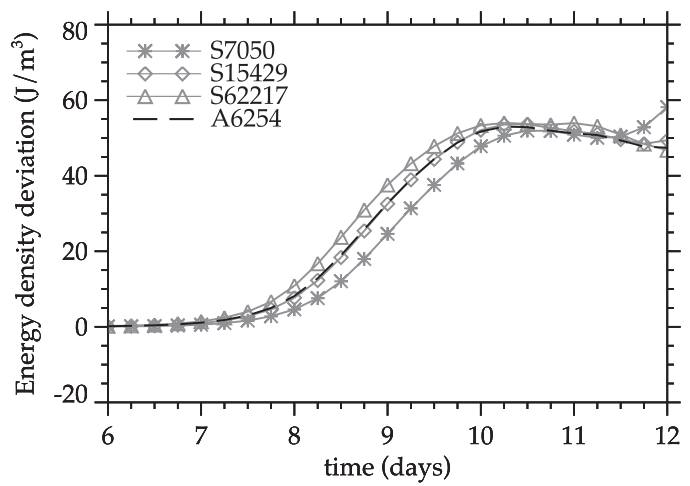

Fig. 6. Time series of the integral kinetic energetics for the different simulations given in Table 2 . Drawn are deviations from the initial value at $t=0$ of $(a)$ total kinetic energy $\langle\mathrm{KE}\rangle$, (b) zonal kinetic energy $\langle\mathrm{ZKE}\rangle$, and (c) eddy kinetic energy $\langle\mathrm{EKE}\rangle$ in the range of day 6 to 12 of the simulations.

domain average; see Appendix D for the respective definitions. Fig. 6 displays deviations of the statistics from their initial values for the simulations specified in Table 2 . The basic behaviour common to all simulations is the increase of $\langle\mathrm{KE}\rangle$ due to the conversion of the ambient state's potential energy in the baroclinic instability process. The large amplitude growth of the baroclinic wave that sets in after 6-7 days is associated with the increase of the eddy kinetic energy $\langle\mathrm{EKE}\rangle$. The growth of $\langle\mathrm{EKE}\rangle$ extracts the potential energy from the baroclinic ambient state, but also (to a lesser extent) from the reservoir of the zonal kinetic energy $\langle Z K E\rangle$, as indicated by the negative values of $\langle Z K E\rangle$. Apart from this commonality, the results exhibit significant differences between the various simulations. The reference simulations S7050, S15429, and S62217 generally pre-

Table 3

Kinetic energetics error norms for various mesh refinement indicators. First column lists simulations as in Table 2, with additional indexing a,b,c,... of the adaptive simulations referring to the applied mesh refinement indicator $\Phi$ specified in the second column. Third, fourth, and fifth column provide error norms $\mathcal{E}_{\langle\mathrm{KE}\rangle}, \mathcal{E}_{\langle\mathrm{ZKE}\rangle}$, and $\mathcal{E}_{\langle\mathrm{EKE}\rangle}$ according to (44) computed as differences in $\langle\mathrm{KE}\rangle,\langle\mathrm{ZKE}\rangle$, and $\langle\mathrm{EKE}\rangle$ between each simulation and the high-resolution reference run S62217.

\begin{tabular}{|c|c|c|c|c|}
\hline Simulation & Refinement indicator $\Phi(t, x, y)$ & $\mathcal{E}_{\langle\mathrm{KE}\rangle}$ & $\mathcal{E}_{\langle\mathrm{ZKE}\rangle}$ & $\mathcal{E}_{\langle\mathrm{EKE}\rangle}$ \\
\hline S7050 & - & 6.43 & 4.99 & 4.84 \\
\hline S15429 & - & 2.58 & 1.66 & 1.90 \\
\hline A6254a & $\frac{1}{H} \int_{0}^{H}\left\|\nabla_{h} \theta\right\| d z$ & 2.82 & 1.67 & 1.80 \\
\hline A6254b & $\left\|\nabla_{h} \theta(z=600 \mathrm{~m})\right\|$ & 3.75 & 2.64 & 2.28 \\
\hline A6254c & $\left\|\nabla_{h} \theta(z=3000 \mathrm{~m})\right\|$ & 2.91 & 1.57 & 1.92 \\
\hline A6254d & $\left\|\nabla_{h} \theta(z=5100 \mathrm{~m})\right\|$ & 2.98 & 2.43 & 1.98 \\
\hline A6254e & $\frac{1}{H} \int_{0}^{H}\|\nabla \times \mathbf{v}\| d z$ & 2.90 & 2.10 & 1.83 \\
\hline A6254f & $\frac{1}{H} \int_{0}^{H}|P V| d z$ & 3.81 & 2.31 & 2.45 \\
\hline A6254g & $|P V(z=5100 \mathrm{~m})|$ & 4.65 & 2.48 & 2.97 \\
\hline A6254h & $|P V(z=9000 \mathrm{~m})|$ & 4.22 & 2.62 & 2.64 \\
\hline A6254i & $\frac{1}{H} \int_{0}^{H}\left\|\nabla_{h} P V\right\| d z$ & 3.82 & 2.36 & 2.65 \\
\hline A6254j & $\frac{1}{H} \int_{0}^{H}|P V| d z, \frac{1}{H} \int_{0}^{H}\left\|\nabla_{h} P V\right\| d z$ & 3.84 & 2.27 & 2.52 \\
\hline A6254k & $\frac{1}{H} \int_{0}^{H}|E P V| d z$ & 10.77 & 5.43 & 8.57 \\
\hline A6254l & $|E P V(z=5100 \mathrm{~m})|$ & 9.50 & 4.60 & 7.56 \\
\hline
\end{tabular}


dict an earlier onset and a faster growth of the instability at higher horizontal resolution. The onset of the major growth of the instability in 57050 is about 0.5 days delayed in comparison to 562217 . After 12 days of model integration, the difference of $\langle\mathrm{KE}\rangle$ between $\mathrm{S} 7050$ and $\mathrm{S} 62217$ is about $12 \mathrm{~J} \mathrm{~m}^{-3}$, a difference of about $20 \mathrm{~J} \mathrm{~m}^{-3}$ is observed for $\langle$ ZKE $\rangle$. The differences between S7050 and S15429 are larger than between S15429 and S62217, suggesting convergence of the statistics. The results obtained with the adaptive solver in A6254 show close agreement with the reference simulation S15429 over the entire integration period.

\subsection{Sensitivity to mesh-refinement indicator}

Table 3 summarises error measures for an extensive series of simulations applying various mesh refinement indicators in the adaptive solver. Shown are $L_{2}$-type error norms based on the difference of $\langle\mathrm{KE}\rangle$, $\langle\mathrm{ZKE}\rangle$, and $\langle$ EKE $\rangle$ between the individual simulations and the high-resolution reference run $\$ 62217$ according to

$$
\mathcal{E}_{\vartheta}=\left(\frac{1}{N_{o}} \sum_{i=1}^{N_{o}}\left(\vartheta_{i}-\vartheta_{i}^{R}\right)^{2}\right)^{1 / 2} \forall \quad \vartheta=\langle\mathrm{KE}\rangle,\langle\mathrm{ZKE}\rangle,\langle\mathrm{EKE}\rangle .
$$

Here $i$ numbers the model outputs in 6 hourly intervals, $N_{o}=48$ is the total number of output times over the full integration period of 12 days, and the superscript $R$ refers to the respective values from the high-resolution reference simulation. In accordance with Fig. 6, the results in Table 3 for A6254a using the mesh refinement indicator (43) are comparable to the reference simulation S15429. The simulation A6254a shows a $2-3$ times reduction in $\mathcal{E}_{\langle\mathrm{KE}\rangle}, \mathcal{E}_{\langle\mathrm{ZKE}\rangle}$, and $\mathcal{E}_{\langle\mathrm{EKE}\rangle}$ over the reference simulation S7050. The other refinement indicators in Table 3 are the horizontal gradient of potential temperature $\nabla_{h} \theta$ at individual model levels $(z=600 \mathrm{~m}, z=3000 \mathrm{~m}$, and $z=5100 \mathrm{~m})$ in A6254b-d, the vertical average of vorticity $\nabla \times \mathbf{v}$ in A6254e, the vertical average of potential vorticity $P V$ defined as

$$
P V=\rho_{b}^{-1}(\nabla \times \mathbf{v}) \cdot \nabla \theta
$$

in A6254f, the PV on two individual model levels ( $z=5100 \mathrm{~m}$ and $z=9000 \mathrm{~m}$ ) in A6254g-h, the vertical average of the horizontal gradient of $P V$ in A6254i, the combination of two refinement indicators $\Phi_{1}$ and $\Phi_{2}$ consisting of the vertical average of $P V$ and $\nabla_{h} P V$ in $A 6254 \mathrm{j}$, the vertical average of the so-called Ertel potential vorticity EPV given as

$$
E P V=\rho_{b}^{-1}(f \mathbf{k}+\nabla \times \mathbf{v}) \cdot \nabla \theta
$$

in $A 6254 \mathrm{k}$, and the EPV at $z=5100 \mathrm{~m}$ in A6254l. In the simulations where more than one mesh refinement indicator $\Phi$ is applied (e.g. A6254j), the individual components of the weighting function (19) are added in a balanced manner adopting suitable normalisation [51,55]. All mesh refinement indicators in Table 3 have a direct association to the physical processes in the baroclinic wave evolution. ${ }^{8}$ In particular, potential vorticity is a fundamental quantity in the dynamics of baroclinic instability [65]. The computed results now reveal that from the tested criteria $\Phi$ in Table 3 , the specification (43) in the configuration A6254a outperforms all other specifications. The largest errors are observed with $\Phi \sim E P V$ in A6254k, substantially larger than with the low-resolution reference simulation S7050. Interestingly, the errors with A6254k are considerably larger than with A6254f using $\Phi \sim P V$. It turned out that the EPV (46) incorporating the Coriolis parameter $f$ puts more emphasis on the vertical component of $\nabla \theta$ (i.e. the thermal stability) compared to $P V$. As a consequence, this larger emphasis on thermal stability leads to a quasi-steady uniform spacing of the mesh in the meridional direction $y$ over the entire simulation, whereas other indicators $\Phi$ show a strongly variable mesh in $y$ (not shown). A further important finding from Table 3 is that the vertically averaged quantities of $\nabla_{h} \theta$ in A6254a and $P V$ in A6254f always provide more accurate results than the corresponding quantities taken at individual model levels in A6254b-d and A6254g-h, respectively.

\subsection{Multiscale performance of the solver}

Imbalances and their spontaneous adjustment in evolving synoptic-scale baroclinic flows are important sources of internal gravity (buoyancy) waves [66]. The generation and propagation of internal gravity waves in complex three-dimensional baroclinic flows is an area of active research, and the interested reader is referred to the literature [66] for discussions of the intricate mechanisms underlying the wave formation. Here, only the representation of mesoscale gravity waves in the simulated baroclinic wave life cycle is addressed. For this purpose, yet another set of simulations is considered, with model configurations summarised in Table 4. Compared to the reference simulations specified in Table 2, an increased vertical resolution with grid interval $\delta \bar{z}=200 \mathrm{~m}$ and a higher horizontal resolution in the adaptive solver are applied. Up to 120 parallel processors are used in the present calculations.

Fig. 7 displays the predicted vertical velocity field $w$ during the later stages of the baroclinic wave development. The data is shown in a portion of the full domain that defines the stratospheric region above the major cyclonic development in the troposphere below, cf. Fig. 5. The conspicuous alternating pattern of positive and negative values in the $w$ field represents a mesoscale internal gravity wave packet with the phase lines oriented roughly from the north-west to the south-east. Table 5

\footnotetext{
${ }^{8}$ Our limited experimentation with indicators based on estimation of local truncation errors [20], corroborates the efficacy of the physics driven mesh refinement.
} 
Table 4

Specifications of the gravity wave simulations. The vertical grid increment is fixed at $\delta \bar{z}=200 \mathrm{~m}$ in all simulations. The time step $\delta \bar{t}$ is selected for each run to give a maximum Courant number of $\mathcal{C}_{\max } \approx 0.5$. All other parameters are as described in Table 2.

\begin{tabular}{llll}
\hline Simulation & $\mathrm{N}_{x} \times \mathrm{N}_{y} \times \mathrm{N}_{z}$ & $\delta \bar{x} \times \delta \bar{y}$ & $\mathrm{~T}_{\mathrm{w}}$ \\
\hline S23213 & $168 \times 140 \times 91$ & $60 \mathrm{~km} \times 60 \mathrm{~km}$ & $240 \mathrm{~s}$ \\
S54967 & $264 \times 210 \times 91$ & $38 \mathrm{~km} \times 38 \mathrm{~km}$ & $1077 \mathrm{~s}$ \\
A21340 & $221 \times 98 \times 91$ & $45 \mathrm{~km} \times 82 \mathrm{~km}$ & $3340 \mathrm{~s}$ \\
\hline
\end{tabular}

(a) S23213

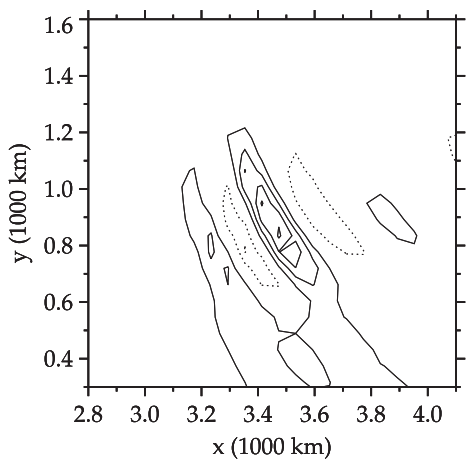

(b) S54967

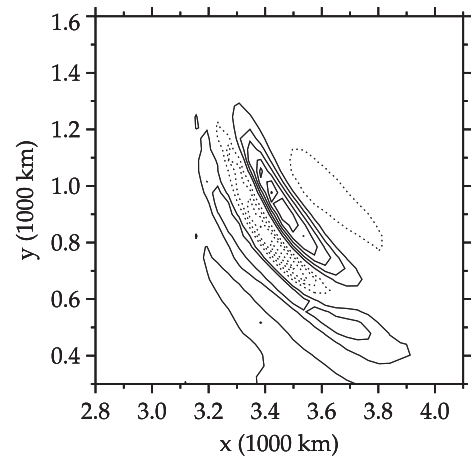

(c) A21340

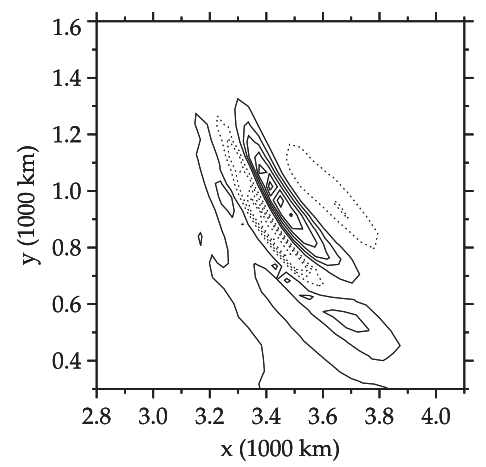

Fig. 7. Horizontal cross section of the vertical velocity field $w\left(\mathrm{~m} \mathrm{~s}^{-1}\right.$, solid contour lines positive, dotted contour lines negative, $\Delta w=0.02 \mathrm{~m} \mathrm{~s}{ }^{-1}$, zero contour line is not shown) at the height $z=12 \mathrm{~km}$ after $t=246 \mathrm{~h}$ of integration time, depicted in a fraction of the full domain. Comparison of the different simulations (a) S23213, (b) S54967, and (c) A21340 (see Table 4).

Table 5

Estimated parameters of the mesoscale internal gravity wave packet shown in Fig. 7 at time $t=246 \mathrm{~h}$. Second and third column: Amplitude given as the maximum and minimum value in the vertical velocity $w$. Fourth and fifth column: Horizontal $\lambda_{h}$ and vertical $\lambda_{z}$ wavelengths.

\begin{tabular}{llll}
\hline Simulation & $w_{\max }$ & $w_{\min }$ & $\lambda_{h}$ \\
\hline S23213 & $0.08 \mathrm{~m} \mathrm{~s}^{-1}$ & $-0.04 \mathrm{~m} \mathrm{~s}^{-1}$ & $290 \mathrm{~km}$ \\
S54967 & $0.12 \mathrm{~m} \mathrm{~s}^{-1}$ & $-0.09 \mathrm{~m} \mathrm{~s}^{-1}$ & $220 \mathrm{~km}$ \\
A21340 & $0.13 \mathrm{~m} \mathrm{~s}^{-1}$ & $-0.09 \mathrm{~m} \mathrm{~s}^{-1}$ & $3.5 \mathrm{~km}$ \\
\hline
\end{tabular}

summarises the estimated parameters of the wave packet for each model configuration. A comparison between the reference simulations S23213 and S54967 shows that the amplitude is considerably smaller and the horizontal wavelength $\lambda_{h}$ much larger in S23213. The internal gravity waves exist near the cut-off of the applied numerical mesh and are not adequately resolved. An observed dominant wavelength $\lambda_{h} \approx 240 \mathrm{~km}$ is covered with about 4 (6) grid points in the run S23213 (S54967). The varying characteristics of the internal gravity wave packet under different resolutions are typical and were observed similarly in other studies, e.g. [60,67,61]. A vertical wavelength of $\lambda_{z}=3.5 \mathrm{~km}$ was estimated for all simulations in Table 5 from vertical cross sections (not shown). In light of the vertical mesh spacing $\delta z=200$ m, this vertical wavelength can be regarded as well resolved. The results in Fig. 7c and Table 5 for the adaptive simulation A21340 - using the refinement indicator $\Phi$ defined in (43) - indicate that the mesoscale internal gravity wave packet is accurately represented. The characteristics of the wave packet in A21340 closely match the characteristics of the high-resolution reference simulation S54967.

\section{Summary}

An atmospheric flow solver with a solution-adaptive moving mesh capability was presented in detail. The solver is based on proven semi-implicit non-oscillatory forward-in-time numerics for the underlying anelastic equations in conservation form [12,9]. To enable mesh adaptivity, the anelastic equations are cast in time-dependent generalised coordinates [8]. The solution-adaptivity of the mesh is implemented by means of moving mesh partial differential equations [10].

Special attention was given to the compatibility of the flux-form advective transport with the anelastic mass continuity. An enhancement to the MPDATA advection scheme $[12,13]$ that achieves full compatibility with mass continuity under arbitrary moving meshes was presented. In addition, to satisfy the geometric conservation law (GCL), required for compatibility, a diagnostic approach based on the treatment of the GCL as an elliptic problem was developed. As demonstrated, ensuring compatibility is essential to provide the monotonicity of advection. Among others, this is important for the implicit large- 
eddy simulation of high-Reynolds number flows. Furthermore, the proposed diagnostic approach for the GCL can be more accurate than the prognostic approach for the GCL [25] established in computational fluid dynamics.

The adaptive solver was first evaluated using the scalar advection test problem of [57]. A straightforward mesh refinement indicator proportional to the gradient of the transported scalar field was employed. In spite of a low number of mesh cells used, the adaptive solver was able to resolve the filamentary structures developing in the scalar field. The available error norms for the adaptive solver were smaller than with a static uniform mesh computation using a factor of 24 larger number of mesh cells.

Next, the adaptive solver was employed to simulate the life cycle of an atmospheric baroclinic instability - an archetype of planetary weather. Intricacies of this flow problem include stratification/rotation effects and nonlinear processes generating a broad motion spectrum. The efficacy of the adaptive moving mesh solver was demonstrated. An improved representation of the flow compared to static uniform mesh computations of similar computational effort were obtained. The latter applies to statistics of the large-scale flow evolution as well as to localised mesoscale features of fronts and internal gravity waves. Typical savings in computational resources through the use of the adaptive solver versus the solver using a uniform mesh were about a factor of 2 to 3 . A crucial aspect of the adaptive simulations is the choice of the mesh refinement indicator. A number of physically motivated indicators were tested. The vertical average of the horizontal gradient of potential temperature (under the Euclidean norm) turned out to be a reliable choice to drive the horizontal mesh adaptation in the three-dimensional flow of the baroclinic wave evolution.

\section{Acknowledgements}

Continuous support from Ulrich Schumann, and discussions with Joseph Prusa are gratefully acknowledged. In addition, we appreciate comments from George Craig, Takashi Misaka, and two referees. This work was funded in part by Deutsche Forschungsgemeinschaft through the MetStröm Priority Research Program (SPP 1276), the DOE award DE-FG0208ER64535, the NSF grant OCI-0904599, and the NCAR visitor programs. Computational resources were provided by the Deutsches Klimarechenzentrum (DKRZ) Hamburg in the project “Multiskalensimulationen mit EULAG". NCAR is sponsored by the National Science Foundation.

\section{Appendix A. Advective velocity prediction in the solution-adaptive moving mesh FT solver}

As addressed in Section 4, the NFT advective transport scheme MPDATA requires an $\mathcal{O}\left(\delta \bar{t}^{2}\right)$ estimate of the generalised contravariant mass flux vector $\left(\rho^{*} \overline{\mathbf{v}}^{*}\right)^{n+1 / 2}$ at the intermediate time level $\bar{t}^{n+1 / 2}$. In our model, the following procedure is applied for the computation: After the adaptation of the mesh coordinates $\mathbf{x}$ from $\bar{t}^{n}$ to $\bar{t}^{n+1}$, the mesh velocity $\mathbf{v}^{g}$ in physical space $\mathbf{S}_{p}$ is computed as

$$
\left(\mathbf{v}^{g}\right)^{n+1 / 2}=\left(\mathbf{x}^{n+1}-\mathbf{x}^{n}\right) / \delta \bar{t} .
$$

Then, $\left(\overline{\mathbf{v}}^{g}\right)^{n+1 / 2}$ in the transformed space $\mathbf{S}_{t}$, the elements of the Jacobian matrix $\widetilde{\mathbf{G}}^{n+1 / 2}$, and the generalised density $\rho^{* n+1 / 2}$ can be obtained from the Kronecker delta relations (5) and the mesh at $\bar{t}^{n+1 / 2}$ approximated by $\mathbf{x}^{n+1 / 2}=0.5\left(\mathbf{x}^{n+1}+\mathbf{x}^{n}\right) .{ }^{9}$ This yields an $\mathcal{O}\left(\delta \bar{t}^{2}\right)$ estimate for $\left(\rho^{*} \overline{\mathbf{v}}^{g}\right)^{n+1 / 2}$ at $\bar{t}^{n+1 / 2}$. The solenoidal mass flux $\left(\rho^{*} \overline{\mathbf{v}}^{s}\right)^{n+1 / 2}$ is obtained from the application of linear or nonlinear predictor schemes [12]. The linear extrapolation scheme, which is employed exclusively in the present work, is given as

$$
\left(\rho^{*} \overline{\mathbf{v}}^{s}\right)^{n+1 / 2}=(1+\beta)\left(\rho^{*} \overline{\mathbf{v}}^{s}\right)^{n}-\beta\left(\rho^{*} \overline{\mathbf{v}}^{s}\right)^{n-1},
$$

where $\beta:=0.5\left(\bar{t}^{n+1}-\bar{t}^{n}\right) /\left(\bar{t}^{n}-\bar{t}^{n-1}\right)$ accounts for a variable time step [68]. After that, the contravariant mass flux $\left(\rho^{*} \overline{\mathbf{v}}^{*}\right)^{n+1 / 2}$ is computed at $\bar{t}^{n+1 / 2}$ as

$$
\left(\rho^{*} \overline{\mathbf{v}}^{*}\right)^{n+1 / 2}=\left(\rho^{*} \overline{\mathbf{v}}^{s}\right)^{n+1 / 2}+\left(\rho^{*} \overline{\mathbf{v}}^{g}\right)^{n+1 / 2},
$$

which completes the procedure. An important aspect is that the described procedure achieves compliance with the $s=0$ component of the GCL identity (6) to machine accuracy for time-dependent coordinate transformations of the one-dimensional form $\mathcal{F}(t, \mathbf{x})=(\bar{x}(t, x), \bar{y}(t, y), \bar{z}(t, z))$ in (1). This property is due to the centred evaluation of the mesh velocity and all metric terms with respect to $\bar{t}^{n}$ and $\bar{t}^{n+1}$, plus the commutativity of the centred spatial derivatives used in the computation of the metric terms. Although the compliance does not hold for more general coordinate mappings, the procedure provides minimisation of the errors with respect to the $s=0$ component of the GCL identity (6) also for arbitrary time-dependent coordinate transformations in (1). Moreover, with the purely two-dimensional mesh adaptation considered in the present work, the $s=1,2,3$ components of the GCL identity (6) are always satisfied to machine accuracy.

\section{Appendix B. Asymptotic accuracy of MPDATA under a moving mesh}

To substantiate the discussion in Section 4.3, elementary numerical tests are presented that investigate the asymptotic accuracy of MPDATA [21] under a moving mesh. The one-dimensional advective conservation law (21) with $\rho_{b} \equiv 1$ and

\footnotetext{
${ }^{9}$ Straightforward averaging of all metric terms and $\rho^{*}$ between $\bar{t}^{n}$ and $\bar{t}^{n+1}$ is equally possible.
} 
(a)

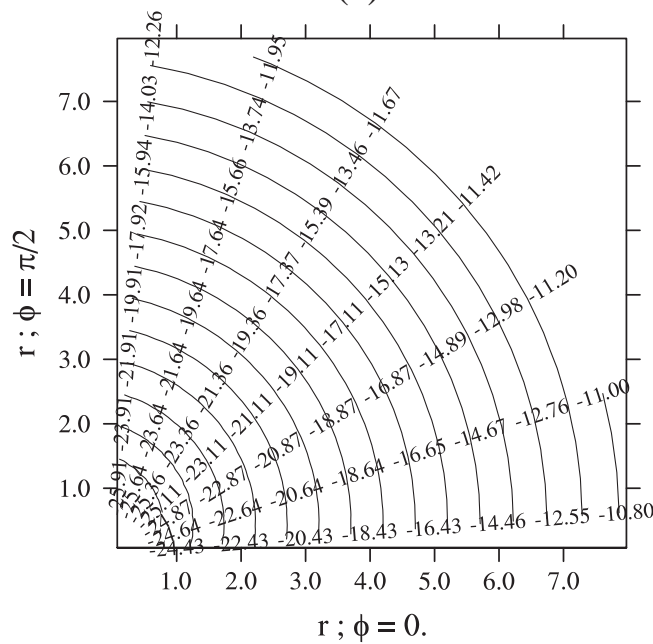

(b)

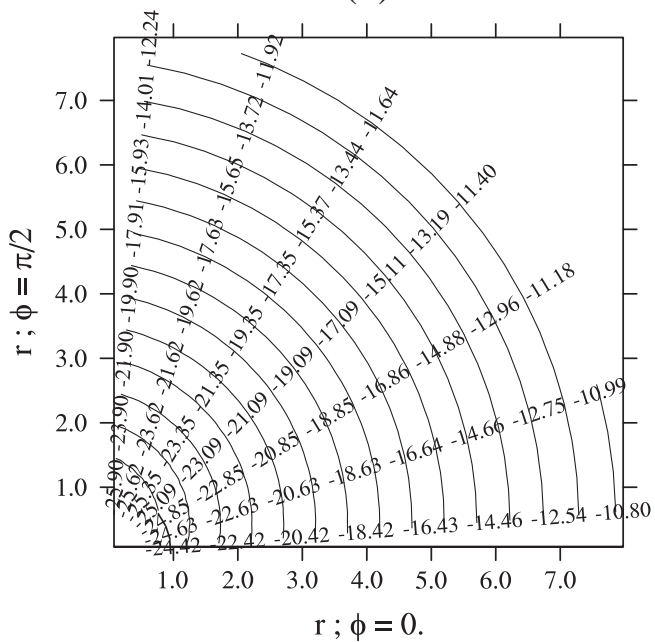

Fig. 8. Asymptotic accuracy of the basic MPDATA under an oscillating mesh; panels (a) and (b) correspond to the original ( 30 ) and the redefined functional form (36) of the pseudo-velocity. Isolines of $\log _{2}(E)$ are shown in a polar system of coordinates that maps the varying spatial resolutions $\delta \bar{x}$ and Courant numbers $\mathcal{C}_{\max }$ upon the radius $r=\log _{2}\left(\delta \bar{x} / \delta \bar{x}_{8}\right)+9$ and the polar angle $\phi=\mathcal{C}_{\max }(\pi / 2)$, respectively. Also given are rays of numerical values of $\log _{2}(E)$ along the radius $r$ at constant Courant numbers $\mathcal{C}_{\max } \in(0.05,0.20,0.35,0.50,0.65,0.80,0.95)$.

$u \equiv 1$ is solved in a domain $0 \leqslant x \leqslant 20$, subject to periodic boundary conditions, and $0 \leqslant t \leqslant T$. An oscillating mesh with periodically changing resolution around the centre of the domain is analytically prescribed using the mapping function ${ }^{10}$

$$
X\left(\bar{X}, S_{f}\right)=1 / 7\left\{\bar{X}\left(15-8 S_{f}^{-1}\right)-\bar{X}^{3}\left(1-S_{f}^{-1}\right)(80-\bar{X}(120-48 \bar{X}))\right\},
$$

where $0 \leqslant X, \bar{X} \leqslant 1$ are normalised physical and transformed coordinates, respectively. The inverse mesh stretching factor is specified as $S_{f}^{-1}(t)=1.0-\gamma \sin ^{2}\left(2 \pi t / T_{0}\right)$, with $\gamma=0.75$ and an oscillation period $T_{0}=10$. The initial condition of $(21)$ is the Gaussian distribution $\psi(0, x)=(1 /(\sigma \sqrt{2 \pi})) \exp \left(-\left(x-x_{0}\right)^{2} / 2 \sigma^{2}\right)$, with $\sigma=2, x_{0}=10$. An integral measure of the error is obtained at time $T$ by means of

$$
E\left(\mathcal{C}_{\max }, \delta \bar{x}\right)=\frac{1}{T}\left(\sum_{i=1}^{\mathrm{N}_{x}}\left(\psi_{E}\left(T, x_{i}\right)-\psi\left(T, x_{i}\right)\right)^{2} / \mathrm{N}_{x}\right)^{1 / 2},
$$

where $\psi_{E}\left(T, x_{i}\right)$ and $\psi\left(T, x_{i}\right)$ are the analytical and numerical solution at position $x_{i}$. For each of the MPDATA formulations OS and RS in Table 1, the error $E\left(\mathcal{C}_{\max }, \delta \bar{x}\right)$ is determined for a range of (maximum) Courant numbers $0.05 \leqslant \mathcal{C}_{\max } \leqslant 0.95$ with increment $\Delta=0.05$, and eight times successively refined grid increments $\delta \bar{x}$ by a factor of 2 , i. e. $\delta \bar{x}=\left(\delta \bar{x}_{8} / 2^{i}\right)$ and $(i=1,8)$, where $\delta \bar{x}_{8}=0.4$. The resulting error surfaces are displayed in Fig. 8. Numerical values of $\log _{2}(E)$ along rays of constant polar angle $\phi$ asymptotically decrease in increments of $\approx-2$ as the resolution increases from $r=8$ to $r=1$. Because the size of the grid increment $\delta \bar{x}$ is halved for every output of $\log _{2}(E)$ along each ray, it demonstrates $E \sim\left(\delta \bar{x}^{2}, \delta \bar{t}^{2}\right)$ as $\delta \bar{x}, \delta \bar{t} \rightarrow 0$ for both formulations OS and RS.

\section{Appendix C. Definition of the potential temperature ambient field}

The specification is provided of the ambient potential temperature field $\theta_{e}$ used in the baroclinic instability experiment of Section 6. Following [59], the height of the tropopause $H_{T}(y)$ is defined as

$$
H_{T}(y)=H_{T, 0}-\left(\frac{g \Delta \theta}{2 \theta_{0}}\right)\left(\frac{1}{N_{s}^{2}-N_{t}^{2}}\right) \tanh \left[\frac{1}{\delta_{\theta}}\left(\frac{y}{w_{j}}+y_{0}\right)\right] .
$$

The potential temperature field $\theta$ at tropospheric heights is given as

$$
\theta_{t}(y, z)=\theta_{0}+\frac{\theta_{0} N_{t}^{2}}{g} z-\frac{\Delta \theta}{2} \tanh \left[\frac{1}{\delta_{\theta}}\left(\frac{y}{w_{j}}+y_{0}-\kappa z\right)\right], \quad z<H_{T}(y)
$$

\footnotetext{
10 The adopted function belongs to an entire suite of analytical mesh mapping functions that offer applicability for various geometrical configurations and boundary conditions $[8,38]$.
} 
and at stratospheric heights it is

$$
\begin{aligned}
& \theta_{s}(y, z)=\theta_{0}+\frac{\theta_{0} N_{s}^{2}}{g} z-\frac{\theta_{0} H_{T}(y) \xi}{g}\left(N_{s}^{2}-N_{t}^{2}\right) \\
& -\frac{\Delta \theta \xi}{2} \tanh \left[\frac{1}{\delta_{\theta}}\left(\frac{y}{w_{j}}+y_{0}-\kappa H_{T}(y)\right)\right]\left(1+\frac{H_{T}(y)-z}{3}\right), \quad z>H_{T}(y),
\end{aligned}
$$

where $\xi(y, z)=\sin \left[0.5 \pi(24000 \mathrm{~m}-z) /\left(24000 \mathrm{~m}-H_{T}(y)\right)\right]$. The tropospheric and stratospheric Brunt-Väisälä frequencies are $N_{t}=10^{-2} \mathrm{~s}^{-1}$ and $N_{s}=\sqrt{6} N_{t}$, respectively. The parameter $w_{j}=1$ controls the width of the baroclinic zone, and $y_{0}=0$. The inverse meridional slope of the tropospheric potential temperature field is $\kappa=70$. The parameter $\Delta \theta=30 \mathrm{~K}$ is a typical meridional variation of potential temperature over the length scale $\delta_{\theta}=525 \mathrm{~km}$. Base values of the tropopause height and the potential temperature are set to $H_{T, 0}=8 \mathrm{~km}$ and $\theta_{0}=273 \mathrm{~K}$, respectively. The resulting potential temperature field is shown in Fig. 4.

\section{Appendix D. Statistical measures of kinetic energetics}

The definitions of the statistical measures of kinetic energetics used in the model analysis of Section 6.2 and remarks about the procedure to derive the quantities from the computed model results are given for completeness. A first quantity considered is the total kinetic energy defined as

$$
\mathrm{KE}=\frac{1}{2} \rho_{b}\left(u^{2}+v^{2}+w^{2}\right)
$$

The initial baroclinic jet flow in the configuration of Section 6 is invariant in the zonal direction $x$ and $v=w=0$. Therefore, it is convenient to decompose $u$ into a zonally averaged part $\langle u\rangle_{x}$ and the respective deviation $u^{\prime}$ according to

$$
u=\langle u\rangle_{x}+u^{\prime}, \quad\langle u\rangle_{x}=\frac{1}{L_{x}} \int_{0}^{L_{x}} u d x .
$$

A zonal kinetic energy (ZKE) is then defined as

$$
\mathrm{ZKE}=\frac{1}{2} \rho_{b}\langle u\rangle_{x}^{2}
$$

while an associated eddy kinetic energy (EKE), i.e. the kinetic energy in terms of the departures from the zonally averaged flow $\langle u\rangle_{x}$, is defined as

$$
\mathrm{EKE}=\frac{1}{2} \rho_{b}\left(u^{\prime 2}+v^{2}+w^{2}\right)
$$

Finally, volume-averaged measures according to

$$
\langle\vartheta\rangle=\frac{1}{L_{x}} \int_{0}^{L_{x}} \frac{1}{L_{y}} \int_{0}^{L_{y}} \frac{1}{H} \int_{0}^{H} \vartheta d x d y d z \quad \forall \vartheta=\mathrm{KE}, \text { ZKE, EKE }
$$

are discussed. The obtained prognostic variables $u, v$ and $w$ on the deformed adaptive mesh are linearly interpolated to a regular uniform mesh in the horizontal at all vertical model levels for the analysis. Thereby, the uniform mesh is chosen twice as fine as the smallest increment of the adaptive mesh, in order to maintain the formal second-order accuracy of the computations. The interpolation procedure accounts for the mesh deformations along the periodic domain boundaries in the streamwise direction.

\section{References}

[1] J. Wu, J.Z. Zhu, J. Szmelter, O.C. Zienkiewicz, Error estimation and adaptivity in Navier-Stokes incompressible flows, Comput. Mech. 6 (1990) 259-270.

[2] R.A. Anthes, Numerical experiments with a two-dimensional horizontal variable grid, Mon. Weather Rev. 98 (1970) $810-822$.

[3] T.L. Clark, R.D. Farley, Severe downslope windstorm calculations in two and three spatial dimensions using anelastic interactive grid nesting: a possible mechanism for gustiness, J. Atmos. Sci. 41 (1984) 329-350.

[4] D.P. Bacon, N.N. Ahmad, Z. Boybeyi, T.J. Dunn, M.S. Hall, P.C.S. Lee, R.A. Sarma, M.D. Turner, K.T. Waight, S.H. Young, J.W. Zack, A dynamically adapting weather and dispersion model: the operational multiscale environment model with grid adaptivity (OMEGA), Mon. Weather Rev. 128 (2000) 20442076.

[5] J. Szmelter, P. Smolarkiewicz, An edge-based unstructured mesh framework for atmospheric flows, Comput. Fluids 46 (2011) $455-460$.

[6] J. Behrens, Adaptive Atmospheric Modeling: Key Techniques in Grid Generation, Data Structures, and Numerical Operations with Applications, Springer, Berlin, 2006.

[7] H. Weller, T. Ringler, M. Piggott, N. Wood, Challenges facing adaptive mesh modeling of the atmosphere and ocean, B. Am. Meteorol. Soc. 91 (2010) $105-108$.

[8] J.M. Prusa, P.K. Smolarkiewicz, An all-scale anelastic model for geophysical flows: dynamic grid deformation, J. Comput. Phys. 190 (2) (2003) 601-622.

[9] J.M. Prusa, P.K. Smolarkiewicz, A.A. Wyszogrodzki, EULAG, a computational model for multiscale flows, Comput. Fluids 37 (2008) $1193-1207$.

[10] W. Huang, Practical aspects of formulation and solution of moving mesh partial differential equations, J. Comput. Phys. 171 (2) (2001) 753-775.

[11] M.A. Taylor, J. Edwards, A. St. Cyr, Petascale atmospheric models for the community climate system model: new developments and evaluation of scalable dynamical cores, J. Phys. Conf. Ser. 125 (2008). 
[12] P.K. Smolarkiewicz, L.G. Margolin, MPDATA: a finite-difference solver for geophysical flows, J. Comput. Phys. 140 (2) (1998) $459-480$.

[13] P.K. Smolarkiewicz, Multidimensional positive definite advection transport algorithm: an overview, Int. J. Numer. Meth. Fl. 50 (2006) 1123-1144.

[14] W.J. Rider, The relationship of MPDATA to other high-resolution methods, Int. J. Numer. Meth. Fl. 50 (2006) 1145-1158.

[15] J.A. Domaradzki, Z. Xiao, P.K. Smolarkiewicz, Effective eddy viscosities in implicit large eddy simulations of turbulent flows, Phys. Fluids 15 (2003) 3890-3893.

[16] L.G. Margolin, W.J. Rider, F.F. Grinstein, Modeling turbulent flow with implicit LES, J. Turbul. 7 (2006) 1-27.

[17] Z.P. Piotrowski, P.K. Smolarkiewicz, S.P. Malinowski, A.A. Wyszogrodzki, On numerical realizability of thermal convection, J. Comput. Phys. 228 (2009) 6268-6290.

[18] M. Lesieur, O. Metais, New trends in large-eddy simulations of turbulence, Ann. Rev. Fluid Mech. 28 (1996) 45-82.

[19] H. Schmidt, U. Schumann, Coherent structure of the convective boundary layer derived from large-eddy simulations, J. Fluid Mech. 200 (1989) 511562.

[20] J. Szmelter, P.K. Smolarkiewicz, MPDATA error estimator for mesh adaptivity, Int. J. Numer. Meth. Fl. 50 (2006) $1269-1293$.

[21] P.K. Smolarkiewicz, W. Grabowski, The multidimensional positive definite advection transport algorithm: nonoscillatory option, J. Comput. Phys. 86 (2) (1990) 355-375.

[22] S. Lin, R.B. Rood, Multidimensional flux-form semi-Lagrangian transport schemes, Mon. Weather Rev. 124 (1996) $2046-2070$.

[23] C. Schär, P.K. Smolarkiewicz, A synchronous and iterative flux-correction formalism for coupled transport equations, J. Comput. Phys. 128 (1996) 101120.

[24] E.S. Gross, L. Bonaventura, G. Rosatti, Consistency with continuity in conservative advection schemes for free-surface models, Int. J. Numer. Meth. Fl. 38 (2002) 307-327.

[25] P. Thomas, C. Lombard, Geometric conservation law and its application to flow computations on moving grids, AIAA J. 17 (1979) $1030-1037$.

[26] J.M. Prusa, P.K. Smolarkiewicz, Dynamic grid deformation: continuous mapping approach, in: Proceedings of the ECMWF seminar series on recent developments in numerical methods for atmospheric and ocean modelling. Reading, UK, 2004, pp. $267-283$.

[27] I. Demirdzic, M. Peric, Space conservation law in finite volume calculations of fluid flow, Int. J. Numer. Meth. Fl. 8 (1988) $1037-1050$.

[28] M.R. Visbal, D.V. Gaitonde, On the use of higher-order finite-difference schemes on curvilinear and deforming meshes, J. Comput. Phys. 181 (2002) $155-185$.

[29] Y.J. Chou, O.B. Fringer, Consistent discretization for simulations of flows with moving generalized coordinates, Int. J. Numer. Meth. Fl. 62 (2009) 802826.

[30] W.W. Grabowski, P.K. Smolarkiewicz, A multiscale anelastic model for meteorological research, Mon. Weather Rev. 130 (2002) $939-956$.

[31] P.K. Smolarkiewicz, J.M. Prusa, Towards mesh adaptivity for geophysical turbulence: continuous mapping approach, Int. J. Numer. Meth. Fl. 47 (2005) 789-801.

[32] F.B. Lipps, R.S. Hemler, A scale analysis of deep moist convection and some related numerical calculations, J. Atmos. Sci. 39 (1982) 2192-2210.

[33] N.P. Wedi, P.K. Smolarkiewicz, Extending Gal-Chen and Somerville terrain-following coordinate transformation on time-dependent curvilinear boundaries, J. Comput. Phys. 193 (2004) 1-20.

[34] J. Synge, A. Schild, Tensor Calculus, Dover, Publications, 1978.

[35] P.K. Smolarkiewicz, L.G. Margolin, A.A. Wyszogrodzki, A class of nonhydrostatic global models, J. Atmos. Sci. 58 (2001) $349-364$.

[36] P. Smolarkiewicz, L. Margolin, On forward-in-time differencing for fluids: an Eulerian/semi-Lagrangian non-hydrostatic model for stratified flows, Atmosphere-Ocean Special 35 (1997) 127-152.

[37] J. Prusa, P. Smolarkiewicz, A. Wyszogrodzki, Simulations of gravity wave induced turbulence using 512 PE Cray T3E, Int. J. Appl. Math. Comput. Sci. 11 (2001) 883-897.

[38] J.M. Prusa, W.J. Gutowski, MPDATA and grid adaptivity in geophysical fluid flow models, Int. J. Numer. Meth. Fl. 50 (2006) 1207-1228.

[39] P.K. Smolarkiewicz, L.G. Margolin, On forward-in-time differencing for fluids - extension to a curvilinear framework, Mon. Weather Rev. 121 (1993) 1847-1859.

[40] P.K. Smolarkiewicz, V. Grubisic, L.G. Margolin, On forward-in-time differencing for fluids: stopping criteria for iterative solutions of anelastic pressure equations, Mon. Weather Rev. 125 (1997) 647-654.

[41] S.C. Eisenstat, H.C. Elman, M.H. Schultz, Variational iterative methods for nonsymmetric systems of linear equations, SIAM J. Numer. Anal. 20 (2) (1983) 345-357.

[42] P.K. Smolarkiewicz, C. Temperton, S.J. Thomas, A.A. Wyszogrodzki, Spectral Preconditioners for nonhydrostatic atmospheric models: extreme applications, In: Proceedings of the ECMWF seminar series on recent developments in numerical methods for atmospheric and ocean modelling. Reading, UK, 2004 pp. 203-220.

[43] J.F. Thompson, F.C. Thames, C.W. Mastin, Automatic numerical generation of body-fitted curvilinear coordinate system for field containing any number of arbitrary two-dimensional bodies, J. Comput. Phys. 15 (1974) 299-319.

[44] J.U. Brackbill, J.S. Saltzman, Adaptive zoning for singular problems in two dimensions, J. Comput. Phys. 46 (1982) $342-368$.

[45] W. Huang, R.D. Russell, Moving mesh strategy based on a gradient flow equation for two-dimensional problems, SIAM J. Sci. Comput. 20 (3) (1999) 998-1015.

[46] A.S. Dvinsky, Adaptive grid generation from harmonic maps on Riemannian manifolds, J. Comput. Phys. 95 (1991) $450-476$.

[47] H.D. Ceniceros, T.Y. Hou, An efficient dynamically adaptive mesh for potentially singular solutions, J. Comput. Phys. 172 (2001) 609-639.

[48] A.M. Winslow, Adaptive mesh zoning by the equipotential method, UCID-19062, Lawrence Livermore National Laboratories, Report, 1981.

[49] G. Beckett, J.A. MacKenzie, A. Ramage, D.M. Sloan, Computational solution of two-dimensional unsteady PDEs using moving mesh methods, J. Comput. Phys. 182 (2002) 478-495.

[50] J. Lang, W. Cao, W. Huang, R.D. Russell, A two-dimensional moving finite element method with local refinement based on a posteriori error estimates, Appl. Numer. Math. 46 (1) (2003) 75-94.

[51] A.v. Dam, P.A. Zegeling, Balanced monitoring of flow phenomena in moving mesh methods, Commun. Comput. Phys. 7 (2010) $138-170$.

[52] B.P. Leonard, A.P. Lock, M.K. MacVean, Conservative explicit unrestricted-time-step multidimensional constancy-preserving advection schemes, Mon. Weather Rev. 124 (1996) 2588-2606.

[53] P.K. Smolarkiewicz, J. Szmelter, MPDATA: an edge-based unstructured-grid formulation, J. Comput. Phys. 206 (2) (2005) 624-649.

[54] P. Smolarkiewicz, J. Prusa, Forward-in-time differencing for fluids: simulation of geophysical turbulence, in: D. Drikakis, B.J. Geurts (Eds.), Turbulent Flow Computation, Kluwer Academic Publishers, Dordrecht, 2002, pp. 279-312.

[55] C. Kühnlein, Solution-adaptive moving mesh solver for geophysical flows, Ph.D. thesis, Ludwig-Maximilians-Universität München, 2011.

[56] P.-O. Persson, J. Bonet, J. Peraire, Discontinuous Galerkin solution of the Navier-Stokes equations on deformable domains, Comput. Methods Appl. Mech. Eng. 198 (2009) 1585-1595.

[57] P.N. Blossey, D.R. Durran, Selective monotonicity preservation in scalar advection, J. Comput. Phys. 227 (2008) $5160-5183$.

[58] R. Rotunno, W.C. Skamarock, C. Snyder, An analysis of frontogenesis in numerical simulations of baroclinic waves, J. Atmos. Sci. 51 (1994) 3373-3398.

[59] A.B.G. Bush, W.R. Peltier, Tropopause folds and synoptic-scale baroclinic wave life cycles, J. Atmos. Sci. 51 (1994) $1581-1604$.

[60] F. Zhang, Generation of mesoscale gravity waves in upper-tropospheric jet front systems, J. Atmos. Sci. 61 (2004) $440-457$.

[61] R. Plougonven, C. Snyder, Inertia-gravity waves spontaneously generated by jets and fronts. Part I: Different baroclinic life cycles, J. Atmos. Sci. 64 (2007) 2502-2520.

[62] P.K. Smolarkiewicz, A. Dörnbrack, Conservative integrals of adiabatic Durran's equations, Int. J. Numer. Meth. Fl. 56 (2008) $1513-1519$.

[63] I.M. Held, M.J. Suarez, A proposal for the intercomparison of the dynamical cores of atmospheric general circulation models, B. Am. Meteorol. Soc. 75 (1994) 1825-1830. 
[64] C. Jablonowski, D.L. Williamson, A baroclinic instability test case for atmospheric model dynamical cores, Q. J. Roy. Meteor. Soc. 132 (2006) $2943-2975$.

[65] B.J. Hoskins, M.E. McIntyre, A.W. Robertson, On the use and significance of isentropic potential vorticity maps, Q. J. Roy. Meteor. Soc. 111 (1985) $877-$ 946.

[66] D.C. Fritts, M.J. Alexander, Gravity wave dynamics and effects in the middle atmosphere, Rev. Geophys. 41 (2003).

[67] C. Zülicke, D. Peters, Simulation of inertia gravity waves in a poleward-breaking Rossby wave, J. Atmos. Sci. 63 (2006) 3253-3276.

[68] P.K. Smolarkiewicz, J. Szmelter, Iterated upwind schemes for gas dynamics, J. Comput. Phys. 228 (2009) 33-54. 